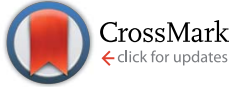

Cite this: RSC Adv., 2015, 5, 69430

\title{
Heteroleptic copper(I) sensitizers with one versus two hole-transporting units in functionalized 2,9- dimethyl-1,10-phenanthroline ancillary ligands $\uparrow$
}

\author{
Sebastian O. Fürer, Biljana Bozic-Weber, Markus Neuburger, Edwin C. Constable \\ and Catherine E. Housecroft*
}

A series of homoleptic $\left[\mathrm{Cu}(\mathrm{L})_{2}\right]\left[\mathrm{PF}_{6}\right]$ complexes in which $\mathrm{L}$ is a 2,9-dimethyl-1,10-phenanthroline fused at the 5,6-positions with a 2'-functionalized imidazole (ligands 1-4), or substituted at the 4,7-positions with electron-donating 4-(diphenylamino)phenyl groups (ligand 5 ) is described; the imidazole 2 '-functionality in 1 is 4-bromophenyl, in 2 is 4-(diphenylamino)phenyl, in 3 is 4-(bis(4-n-butoxy)phenylamino)phenyl, and in 4 is 4-(carbazol-9-yl)phenyl. The copper complexes were characterized by mass spectrometry, NMR and absorption spectroscopies and cyclic voltammetry; the single crystal structure of ligand 4 has been determined. Compared to the solution absorption spectra of $\left[\mathrm{Cu}(1)_{2}\right]\left[\mathrm{PF}_{6}\right],\left[\mathrm{Cu}(2)_{2}\right]\left[\mathrm{PF}_{6}\right],\left[\mathrm{Cu}(3)_{2}\right]\left[\mathrm{PF}_{6}\right]$ and $\left[\mathrm{Cu}(4)_{2}\right]\left[\mathrm{PF}_{6}\right]$, that of $\left[\mathrm{Cu}(5)_{2}\right]\left[\mathrm{PF}_{6}\right]$ shows increased absorbance at wavelengths $>375 \mathrm{~nm}$. An on-surface strategy was used to assemble heteroleptic $[\mathrm{Cu}(6)(\mathrm{L})]^{+}$dyes on $\mathrm{TiO}_{2}$ electrodes where 6 is ((6,6'-dimethyl-[2,2'-bipyridine]-4,4'-diyl)bis(4,1-phenylene))bis(phosphonic acid); solid-state absorption spectra confirmed enhanced light-harvesting between 375 and $600 \mathrm{~nm}$ for $[\mathrm{Cu}(6)(5)]^{+}$with respect to $[\mathrm{Cu}(6)(1)]^{+},[\mathrm{Cu}(6)(2)]^{+},[\mathrm{Cu}(6)(3)]^{+}$and $[\mathrm{Cu}(6)(4)]^{+}$. Comparison of the performances of dye-sensitized solar cells (DSCs) containing $[\mathrm{Cu}(6)(2)]^{+},[\mathrm{Cu}(6)(3)]^{+}$and $[\mathrm{Cu}(6)(4)]^{+}$with those with $[\mathrm{Cu}(6)(1)]^{+}$indicate only a marginal influence of the diphenylamine or carbazole hole-transporting domains in 5,6-substituted phenanthroline dyes. The introduction of the 4-(diphenylamino)phenyl hole-transporting units in the 4- and 7-positions of the phen unit in 5 proves to be beneficial, with DSCs containing $[\mathrm{Cu}(6)(5)]^{+}$performing better than those with the other four dyes; duplicate DSCs were tested for each dye to validate the results. While the values of the maximum external quantum efficiencies $\left(E Q E_{\max }\right)$ for $[\mathrm{Cu}(6)(1)]^{+}$and $[\mathrm{Cu}(6)(4)]^{+}$are greater than for $[\mathrm{Cu}(6)(5)]^{+}$, the extension of the EQE spectrum for $[\mathrm{Cu}(6)(5)]^{+}$to longer wavelengths results in higher short-circuit current densities $\left(J_{S C}\right)$ compared to DSCS with $[\mathrm{Cu}(6)(1)]^{+},[\mathrm{Cu}(6)(2)]^{+},[\mathrm{Cu}(6)(3)]^{+}$and $[\mathrm{Cu}(6)(4)]^{+}$

Received 25th June 2015

Accepted 7th August 2015

DOI: $10.1039 / c 5 r a 12296 a$

www.rsc.org/advances

\section{Introduction}

The development of dye-sensitized solar cells (DSCs) has progressed from the prototype ruthenium dyes of Grätzel and $\mathrm{O}^{\prime}$ Regan, ${ }^{1,2}$ to the use of organic ${ }^{3}$ and porphyrin-containing ${ }^{4}$ dyes with solar-to-electrical power conversion efficiencies (PCEs) reaching $\approx 12 \% .{ }^{5}$ Recently, perovskite DSCs have excited considerable attention, with PCEs of $18-20 \% .^{6-8}$ Our contributions to the advancement of DSCs focus on sustainable components, ${ }^{9}$ in particular with copper-containing dyes ${ }^{10}$ replacing those containing precious metals. The potential of

Department of Chemistry, University of Basel, Spitalstrasse 51, CH-4056 Basel, Switzerland. E-mail: catherine.housecroft@unibas.ch; Tel: +41612671008

$\dagger$ Electronic supplementary information (ESI) available: Fig. S1: representative HMQC and HMBC spectra. Fig. S2: cyclic voltammograms of copper(I) complexes; Fig. S3: photographs of dye-functionalized electrodes; Fig. S4-S6: $J-V$ curves and EQE spectra. CCDC 1405837. For ESI and crystallographic data in CIF or other electronic format see DOI: 10.1039/c5ra12296a
copper(I) dyes was first recognized by Sauvage and coworkers, ${ }^{11}$ and in 2014 , PCEs exceeding $3 \%$ (relative to $\approx 7.5 \%$ for reference dye N719) were achieved. ${ }^{12,13}$ Homoleptic copper(I) complexes have also been used as redox mediators combined with ruthenium(II) sensitizers in DSCs. ${ }^{14}$

The simplest copper(I) sensitizers are homoleptic complexes of type $\left[\mathrm{Cu}\left(\mathrm{L}_{\text {anchor }}\right)_{2}\right]^{+}$in which $\mathrm{L}_{\text {anchor }}$ is typically a diimine ligand bearing a carboxylic or phosphonic acid substituent to anchor the dye to the semiconductor surface. ${ }^{15}$ Dye performance is most easily improved and tuned by employing heteroleptic $\left[\mathrm{Cu}\left(\mathrm{L}_{\text {anchor }}\right)\left(\mathrm{L}_{\text {ancillary }}\right)\right]^{+}$dyes, although these are often difficult to isolate because of the lability of bis(diimine)copper(I) complexes. ${ }^{16}$ Two approaches to access heteroleptic dyes are now successfully used. The first is the HETPHEN strategy ${ }^{17}$ introduced by Odobel and coworkers ${ }^{13,18}$ which relies on bulky ligands to hinder ligand exchange. Using this approach, a remarkable efficiency of $4.66 \%$ (relative to $7.36 \%$ for N719) has been recorded for the dye shown in Scheme 1a in the presence of the co-adsorbant chenodeoxycholic acid. ${ }^{13} \mathrm{~A}$ second route to 
heteroleptic dyes is our 'surface-as-ligand, surface-as-complex' approach ${ }^{\mathbf{1 9 - 2 2}}$ which involves a stepwise assembly of heteroleptic metal complex dyes on electrode surfaces and has been used for both copper(I) ${ }^{22}$ and zinc(II) ${ }^{23}$ sensitizers. The strategy provides a straightforward means for rapid screening of different combinations of anchoring and ancillary ligands. To assemble a $\left[\mathrm{Cu}\left(\mathrm{L}_{\text {anchor }}\right)\left(\mathrm{L}_{\text {ancillary }}\right)\right]^{+}$dye, an electrode is initially soaked in a solution of $\mathrm{L}_{\text {anchor, }}$, and then the functionalized electrode is immersed in a dye-bath containing either $\left[\mathrm{Cu}\left(\mathrm{L}_{\text {ancillary }}\right)_{2}\right]^{+}$or a mixture of $\left[\mathrm{Cu}(\mathrm{MeCN})_{4}\right]^{+}$and $\mathrm{L}_{\text {ancillary. }}{ }^{22,24}$

The incorporation of imidazo $\left[4^{\prime}, 5^{\prime}: 5,6\right]-1,10$-phenanthroline ligands bearing electron-donating groups in the $2^{\prime}$-position has been shown to be advantageous in ruthenium-based sensitizers, ${ }^{25}$ and these ligands are also attractive for copper(I)-based DSCs. ${ }^{18,26}$ The imidazo[ $\left[4^{\prime}, 5^{\prime}: 5,6\right]-1,10$-phenanthroline unit is readily extended with a 4 -(diphenylamino)phenyl ${ }^{18}$ or other holetransporting unit, and Scheme 1b shows a copper(I) sensitizer which is noteworthy for its broad absorption spectrum extending beyond $700 \mathrm{~nm}$; however, DSCs containing this dye gave efficiencies of $<0.3 \%$ (with respect to $6.55 \%$ for N719). ${ }^{18}$ Ligand $1{ }^{26}$ (Scheme 2) is a convenient precursor to $2^{\prime}$-functionalized 2,9dimethyl-imidazo $\left[4^{\prime}, 5^{\prime}: 5,6\right]-1,10$-phenanthrolines for use as ancillary ligands in $\left[\mathrm{Cu}\left(\mathrm{L}_{\text {anchor }}\right)\left(\mathrm{L}_{\text {ancillary }}\right)\right]^{+}$dyes. The 2,9-substituents in the phen metal-binding domain stabilize copper(I) with respect to oxidation by sterically hindering the transformation of tetrahedral copper(I) to square planar copper(II). An additional feature of $\mathbf{1}$ is the long $N$-alkyl substituent which helps to prevent intermolecular aggregation of dye molecules on the semiconductor surface and also militates against charge recombination processes. ${ }^{27}$

We now report the development of heteroleptic copper(I) dyes for DSCs with ancillary ligands derived through postfunctionalization of the peripheral bromo-substituent in $\mathbf{1}$. We also demonstrate the effects of introducing hole-

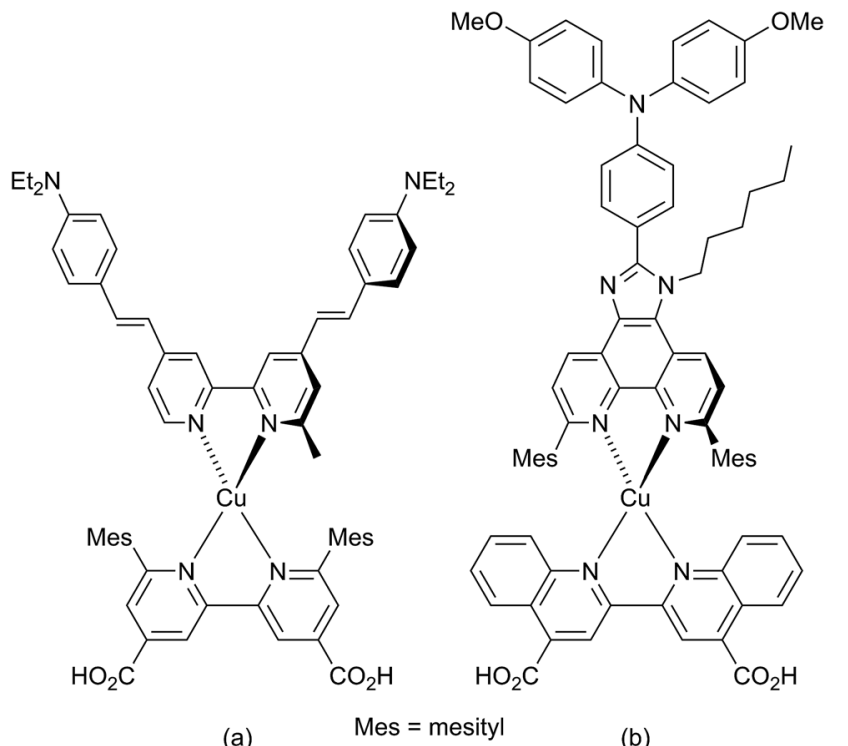

Scheme 1 Copper(I) sensitizers reported by Odobel and coworkers (see text).
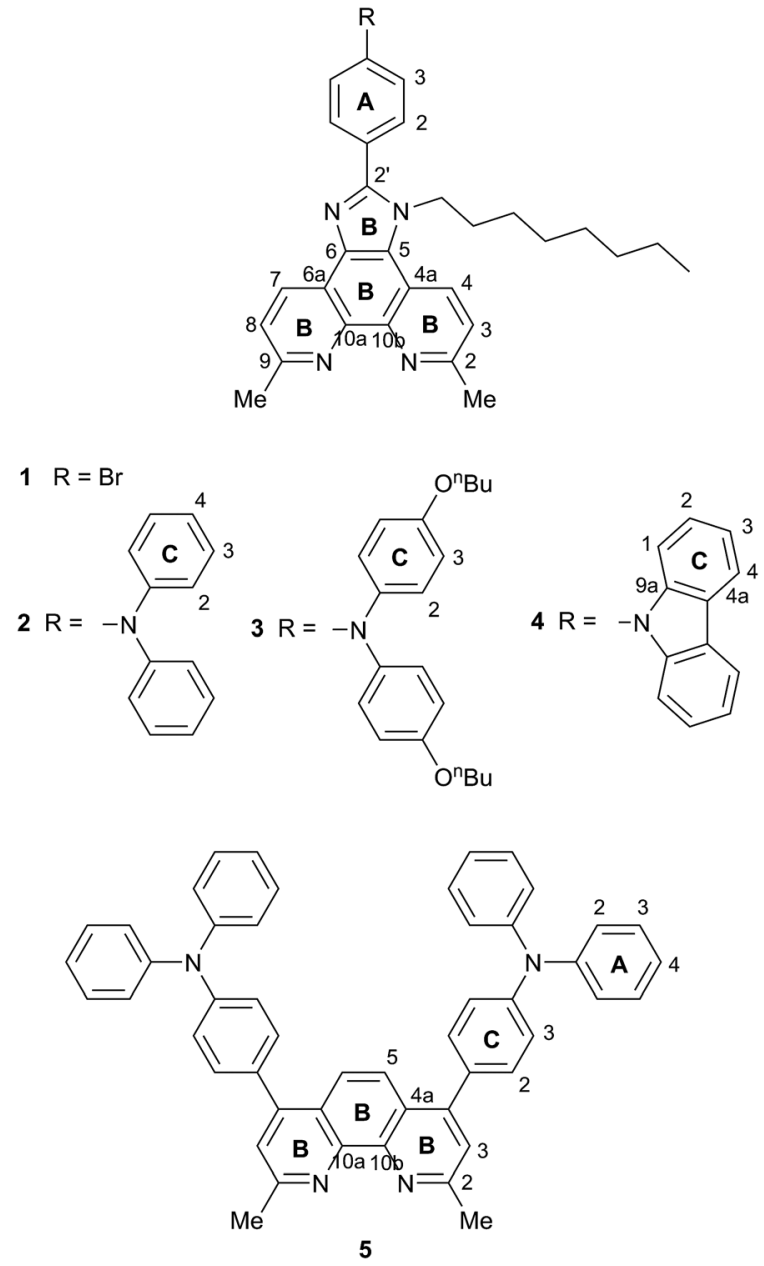

Scheme 2 Structures of ligands 1-5. Ring labelling is for NMR assignments.

transporting domains into the 4- and 7-positions of 2,9-dimethyl-1,10-phenanthroline.

\section{Experimental}

\section{General}

${ }^{1} \mathrm{H}$ and ${ }^{13} \mathrm{C}$ NMR spectra were recorded at $295 \mathrm{~K}$ on a Bruker Avance III-500 NMR spectrometer with chemical shifts referenced to residual solvent peaks with respect to $\delta(\mathrm{TMS})=0 \mathrm{ppm}$. Solution and solid-state absorption spectra were recorded on Perkin-Elmer Lambda 25 and Cary 5000 spectrophotometers, respectively, and FT-IR spectra on a Perkin-Elmer Spectrum Two spectrometer equipped with a UATR. Electrospray (ESI) mass spectra (solution samples in $\mathrm{MeOH}$ with a drop of $\mathrm{CH}_{2} \mathrm{Cl}_{2}$ added) and high resolution ESI-MS were measured on Bruker Esquire $3000^{\text {plus }}$ and Bruker maXis $4 \mathrm{G}$ instruments, respectively.

Electrochemical measurements were performed on a $\mathrm{CHI}$ 900B instrument by cyclic voltammetry (CV) using a glassy carbon working electrode, platinum wire auxiliary electrode, and a silver wire pseudo-reference electrode. HPLC grade, argon degassed $\mathrm{CH}_{2} \mathrm{Cl}_{2}$ solutions $\left(\approx 10^{-4} \mathrm{~mol} \mathrm{dm}{ }^{-3}\right)$ of the copper 
complexes were used with $0.1 \mathrm{M}\left[{ }^{n} \mathrm{Bu}_{4} \mathrm{~N}\right]\left[\mathrm{PF}_{6}\right]$ as supporting electrolyte; the scan rate was $0.1 \mathrm{~V} \mathrm{~s}^{-1}$ and ferrocene was used as an internal standard, added at the end of each experiment.

\section{Ligands and complexes}

Compound $1,{ }^{26} 2{ }^{26}$ 2,9-dimethyl-1,10-phenanthroline-5,6-dione, ${ }^{28}$ 4-(9H-carbazol-9-yl)benzaldehyde, ${ }^{29} \quad$ 4,7-dichloro-2,9-dimethyl1,10-phenanthroline, ${ }^{30}\left[\mathrm{Cu}(\mathrm{MeCN})_{4}\right]\left[\mathrm{PF}_{6}\right]^{31}$ and $\left[\mathrm{Cu}(2)_{2}\right]\left[\mathrm{PF}_{6}\right]^{26}$ were synthesized as previously reported. 4,4'-Di- $n$-butoxydiphenylamine was prepared by the method reported for the analogous hexoxy derivative, ${ }^{32}$ and NMR spectra corresponded to those published..$^{33}$ Bis(dibenzylideneacetone)palladium(0), $\left[\mathrm{Pd}(\mathrm{dba})_{2}\right]$ was purchased from Strem Chemicals, and $4-(N, N-$ diphenylamino)phenylboronic acid from Fluorochem.

Compound 3 a flask (50 ml) was charged with 1 (300 mg, $0.582 \mathrm{mmol}), \quad 4,4^{\prime}$-di- $n$-butoxydiphenylamine $(279 \mathrm{mg}$, $0.873 \mathrm{mmol}$ ), $\mathrm{NaO}^{t} \mathrm{Bu}(140 \mathrm{mg}, 1.45 \mathrm{mmol}$ ) and a catalytic amount of $\left[\mathrm{Pd}(\mathrm{dba})_{2}\right](18.4 \mathrm{mg}, 0.0291 \mathrm{mmol})$ and was evacuated for $15 \mathrm{~min}$. Toluene $(20 \mathrm{ml})$ and $\mathrm{P}^{t} \mathrm{Bu}_{3}(0.03 \mathrm{ml}$ of a $0.1 \mathrm{M}$ sol. in toluene, $23.9 \mathrm{mg}, 0.0291 \mathrm{mmol}$ ) were added and the reddish brown mixture was heated to $95{ }^{\circ} \mathrm{C}$ for 8 days. The mixture was allowed to cool to room temperature, filtered and the solvent was removed. The remaining brown solid was purified by column chromatography on alumina eluting with $\mathrm{CH}_{2} \mathrm{Cl}_{2}$ to yield the product as a yellow solid $(223 \mathrm{mg}, 298 \mathrm{mmol}$, $51.2 \%) .{ }^{1} \mathrm{H}$ NMR (500 MHz, $\mathrm{CDCl}_{3}$ ) $\delta / \mathrm{ppm} 9.24$ (br, $1 \mathrm{H}, \mathrm{H}^{\mathrm{B} 4 / \mathrm{B} 7}$ ), $8.48\left(\mathrm{~d}, J=8.6 \mathrm{~Hz}, 1 \mathrm{H}, \mathrm{H}^{\mathrm{B} 4 / \mathrm{B} 7}\right), 7.66\left(\mathrm{~d}, J=8.2 \mathrm{~Hz}, 1 \mathrm{H}, \mathrm{H}^{\mathrm{B} 3 / \mathrm{B} 8}\right)$, $7.62\left(\mathrm{~d}, J=8.4 \mathrm{~Hz}, 1 \mathrm{H}, \mathrm{H}^{\mathrm{B} 3 / \mathrm{B} 8}\right), 7.54\left(\mathrm{~m}, 2 \mathrm{H}, \mathrm{H}^{\mathrm{A} 2}\right), 7.13(\mathrm{~m}, 4 \mathrm{H}$, $\left.\mathrm{H}^{\mathrm{C} 2}\right), 7.03\left(\mathrm{~m}, 2 \mathrm{H}, \mathrm{H}^{\mathrm{A} 3}\right), 6.88\left(\mathrm{~m}, 4 \mathrm{H}, \mathrm{H}^{\mathrm{C} 3}\right), 4.64\left(\mathrm{~m}, 2 \mathrm{H}, \mathrm{H}^{\mathrm{NCH}_{2}}\right)$, $3.96\left(\mathrm{t}, J=6.5 \mathrm{~Hz}, 4 \mathrm{H}, \mathrm{H}^{\mathrm{OCH}_{2}}\right), 3.07\left(\mathrm{~s}, 3 \mathrm{H}, \mathrm{H}^{\text {Me-phen }}\right), 3.01(\mathrm{~s}, 3 \mathrm{H}$, $\left.\mathrm{H}^{\mathrm{Me}-\mathrm{phen}}\right), 1.95\left(\mathrm{~m}, 2 \mathrm{H}, \mathrm{H}^{\mathrm{NCH}_{2} \mathrm{CH}_{2}}\right), 1.78\left(\mathrm{~m}, 4 \mathrm{H}, \mathrm{H}^{\mathrm{OCH}_{2} \mathrm{CH}_{2}}\right), 1.51$ $\left(\mathrm{m}, 4 \mathrm{H}, \mathrm{H}^{\mathrm{OCH}_{2} \mathrm{CH}_{2} \mathrm{CH}_{2}}\right), 1.30-1.16\left(\mathrm{~m}, 10 \mathrm{H}, \mathrm{H}^{\mathrm{CH}_{2} \text {-octyl }}\right), 0.99(\mathrm{t}, J=$ $\left.7.4 \mathrm{~Hz}, 6 \mathrm{H}, \mathrm{H}^{\text {Me-butyl }}\right), 0.85\left(\mathrm{t}, J=6.9 \mathrm{~Hz}, 3 \mathrm{H}, \mathrm{H}^{\text {Me-octyl }}\right) .{ }^{13} \mathrm{C}$ NMR $\left(126 \mathrm{MHz}, \mathrm{CDCl}_{3}\right) \delta / \mathrm{ppm} 157.5\left(\mathrm{C}^{\mathrm{B} 2+\mathrm{B} 9}\right), 156.1\left(\mathrm{C}^{\mathrm{C} 4}\right), 139.6\left(\mathrm{C}^{\mathrm{C} 1}\right)$, $130.6\left(\mathrm{C}^{\mathrm{A} 2}\right), 128.7\left(\mathrm{C}^{\mathrm{B} 4 / \mathrm{B} 7}\right), 127.6\left(\mathrm{C}^{\mathrm{C} 2}\right), 124.5\left(\mathrm{C}^{\mathrm{B} 3 / \mathrm{B} 8}\right), 123.6$ $\left(\mathrm{C}^{\mathrm{B} 3 / \mathrm{B} 8}\right), 118.6\left(\mathrm{C}^{\mathrm{A} 3}\right), 117.5\left(\mathrm{C}^{\mathrm{B} 4 \mathrm{a} / \mathrm{B} 6 \mathrm{a}}\right), 115.6\left(\mathrm{C}^{\mathrm{C} 3}\right), 67.9\left(\mathrm{C}^{\mathrm{OCH}_{2}}\right)$, $46.9\left(\mathrm{C}^{\mathrm{NCH}_{2}}\right), 31.8\left(\mathrm{C}^{\mathrm{CH}_{2} \text {-octyl }}\right), 31.5\left(\mathrm{C}^{\mathrm{OCH}_{2} \mathrm{CH}_{2}}\right), 30.2,\left(\mathrm{C}^{\mathrm{NCH}_{2} \mathrm{CH}_{2}}\right)$, $29.2\left(\mathrm{C}^{\mathrm{CH}_{2} \text {-octyl }}\right), 29.0\left(\mathrm{C}^{\mathrm{CH}_{2} \text {-octyl }}\right), 26.4\left(\mathrm{C}^{\mathrm{CH}_{2} \text { octyl }}\right), 25.3\left(\mathrm{C}^{\text {phen-Me }}\right)$, $25.1\left(\mathrm{C}^{\text {phen-Me }}\right), \quad 22.7\left(\mathrm{C}^{\mathrm{CH}_{2} \text {-octyl }}\right), \quad 19.4 \quad\left(\mathrm{C}^{\mathrm{OCH}_{2} \mathrm{CH}_{2} \mathrm{CH}_{2}}\right), \quad 14.2$ $\left(\mathrm{C}^{\text {Me-octyl }}\right)$, $14.0\left(\mathrm{C}^{\text {Me-butyl }}\right)$, (other $\mathrm{C}^{\mathrm{Q}}$ not resolved). UV-Vis $\left(\mathrm{CH}_{2} \mathrm{Cl}_{2}, 1.00 \times 10^{-5} \mathrm{~mol} \mathrm{dm}{ }^{-3}\right) \lambda / \mathrm{nm}\left(\varepsilon / \mathrm{dm}^{3} \mathrm{~mol}^{-1} \mathrm{~cm}^{-1}\right)$ 267 (35 700), 296 (40 700), 341 sh (23 800). HR ESI-MS m/z: 748.4582 $[\mathrm{M}+\mathrm{H}]^{+}$(calc. 748.4585). Found $\mathrm{C} 78.52, \mathrm{H} 7.51, \mathrm{~N}$ 8.96; $\mathrm{C}_{49} \mathrm{H}_{57} \mathrm{~N}_{5} \mathrm{O}_{2}$ requires $\mathrm{C} 78.68, \mathrm{H} 7.68, \mathrm{~N} 9.36 \%$.

Compound 4a 4-(9H-carbazol-9-yl)benzaldehyde (535 mg, $1.97 \mathrm{mmol}), \quad$ 2,9-dimethyl-1,10-phenanthroline-5,6-dione (481 $\mathrm{mg}, 2.02 \mathrm{mmol}$ ) and an excess of $\mathrm{NH}_{4} \mathrm{OAc}(5.04 \mathrm{~g}$, $65.4 \mathrm{mmol})$ were added to $\mathrm{EtOH}(100 \mathrm{ml})$. The yellow mixture was heated at reflux overnight after which time the solvent was removed. The remaining orange solid was washed with water and $\mathrm{Et}_{2} \mathrm{O}$. The product was purified using column chromatography (alumina, $\mathrm{CH}_{2} \mathrm{Cl}_{2}$ with $3 \% \mathrm{MeOH}$ ) and was isolated as a yellow solid (282 mg, $0.575 \mathrm{mmol}, 29.2 \%) .{ }^{1} \mathrm{H}$ NMR (500 MHz, DMSO- $\left.d_{6}\right) \delta /$ ppm $13.8(\mathrm{br}, \mathrm{NH}), 8.84\left(\mathrm{~d}, J=8.2 \mathrm{~Hz}, 2 \mathrm{H}, \mathrm{H}^{\mathrm{B} 4}\right), 8.56$ $\left(\mathrm{m}, 2 \mathrm{H}, \mathrm{H}^{\mathrm{A} 2}\right), 8.29\left(\mathrm{~m}, 2 \mathrm{H}, \mathrm{H}^{\mathrm{C} 4}\right), 7.91\left(\mathrm{~m}, 2 \mathrm{H}, \mathrm{H}^{\mathrm{A} 3}\right), 7.72(\mathrm{~m}, 2 \mathrm{H}$, $\left.\mathrm{H}^{\mathrm{B} 3}\right), 7.55\left(\mathrm{~m}, 2 \mathrm{H}, \mathrm{H}^{\mathrm{C} 1}\right), 7.49\left(\mathrm{~m}, 2 \mathrm{H}, \mathrm{H}^{\mathrm{C} 2}\right), 7.34\left(\mathrm{~m}, 2 \mathrm{H}, \mathrm{H}^{\mathrm{C} 3}\right)$, $2.82(\mathrm{~s}, 6 \mathrm{H}) .{ }^{13} \mathrm{C}$ NMR $\left(126 \mathrm{MHz}, \mathrm{DMSO}-d_{6}\right) 156.0\left(\mathrm{C}^{\mathrm{B} 2}\right), 149.3$ $\left(\mathrm{C}^{\text {imid-2 }}\right), 143.1\left(\mathrm{C}^{\mathrm{B} 10 \mathrm{a}}\right), 139.8\left(\mathrm{C}^{\mathrm{C} 9 \mathrm{a}}\right), 137.6\left(\mathrm{C}^{\mathrm{A} 4}\right), 129.9\left(\mathrm{C}^{\mathrm{B} 4}\right)$, $129.0\left(\mathrm{C}^{\mathrm{A} 1}\right), 127.6\left(\mathrm{C}^{\mathrm{A} 2}\right), 126.9\left(\mathrm{C}^{\mathrm{A} 3}\right), 126.5\left(\mathrm{C}^{\mathrm{B} 5}\right), 126.3\left(\mathrm{C}^{\mathrm{C} 2}\right)$, $123.2\left(\mathrm{C}^{\mathrm{B} 3}\right), 120.5\left(\mathrm{C}^{\mathrm{C} 4}\right), 120.2\left(\mathrm{C}^{\mathrm{C} 3}\right), 109.6\left(\mathrm{C}^{\mathrm{C} 1}\right), 24.7\left(\mathrm{C}^{\mathrm{Me}}\right),\left(\mathrm{C}^{\mathrm{B} 4 \mathrm{a}}\right.$ not resolved). ESI MS $m / z: 490.4[\mathrm{M}+\mathrm{H}]^{+}$(calc. 490.2).

Compound $4 \mathrm{NaH}$ (60\% oil dispersion, $122 \mathrm{mg}, 3.05 \mathrm{mmol}$ ) was suspended and stirred vigorously for $1.5 \mathrm{~h}$ in DMF $(5 \mathrm{ml})$ under $\mathrm{N}_{2}$. Compound $4 \mathrm{a}$ (200 $\mathrm{mg}, 0.409 \mathrm{mmol}$ ) was added and the suspension was stirred for $10 \mathrm{~min}$. Then 1-bromo- $n$-octane $(0.711 \mathrm{ml}, 789 \mathrm{mg}, 4.09 \mathrm{mmol})$ was added and the mixture was heated at $70{ }^{\circ} \mathrm{C}$ for 3 days. The black mixture was allowed to cool to room temperature and was diluted with water, giving a suspension which was extracted with $\mathrm{CH}_{2} \mathrm{Cl}_{2}(25 \mathrm{ml})$. The organic layer was washed with water $(5 \times 40 \mathrm{ml})$, dried over $\mathrm{Na}_{2} \mathrm{SO}_{4}$ and the solvent was removed. The remaining DMF was removed by azeotrope distillation with toluene five times. The brown residue was washed with petroleum ether and dried in vacuo. This gave a brown foam which was purified by column chromatography (three columns: alumina, $\mathrm{CH}_{2} \mathrm{Cl}_{2}$ changing to $\mathrm{CH}_{2} \mathrm{Cl}_{2} / 2 \% \mathrm{MeOH} ;$ alumina, toluene/ethyl acetate $4: 1$ changing to 1 : 1 ; alumina, $\left.\mathrm{CH}_{2} \mathrm{Cl}_{2} / 0.25 \% \mathrm{MeOH}\right)$. Compound 4 was isolated as a beige solid (45 mg, $0.075 \mathrm{mmol}, 18.3 \%$ ). ${ }^{1} \mathrm{H}$

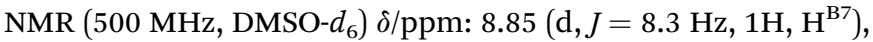
$8.78\left(\mathrm{~d}, J=8.6 \mathrm{~Hz}, 1 \mathrm{H}, \mathrm{H}^{\mathrm{B} 4}\right), 8.30\left(\mathrm{~d}, J=7.9 \mathrm{~Hz}, 2 \mathrm{H}, \mathrm{H}^{\mathrm{C} 4}\right), 8.10$ $\left(\mathrm{m}, 2 \mathrm{H}, \mathrm{H}^{\mathrm{A} 2}\right), 7.91\left(\mathrm{~m}, 2 \mathrm{H}, \mathrm{H}^{\mathrm{A} 3}\right), 7.77\left(\mathrm{~d}, J=8.3 \mathrm{~Hz}, 1 \mathrm{H}, \mathrm{H}^{\mathrm{B} 3}\right)$, $7.73\left(\mathrm{~d}, J=8.0 \mathrm{~Hz}, 1 \mathrm{H}, \mathrm{H}^{\mathrm{B} 8}\right), 7.56\left(\mathrm{~d}, J=8.2 \mathrm{~Hz}, 2 \mathrm{H}, \mathrm{H}^{\mathrm{C} 1}\right), 7.50$ $\left(\mathrm{t}, J=7.6 \mathrm{~Hz}, 2 \mathrm{H}, \mathrm{H}^{\mathrm{C} 2}\right), 7.35\left(\mathrm{t}, J=7.3 \mathrm{~Hz}, 2 \mathrm{H} \mathrm{H}^{\mathrm{C} 3}\right), 4.82(\mathrm{~m}, 2 \mathrm{H}$, $\left.\mathrm{H}^{\mathrm{NCH}_{2}}\right), 2.84$ (s, 3H, H $\left.\mathrm{H}^{\text {Me-phen }}\right), 2.83\left(\mathrm{~s}, 3 \mathrm{H}, \mathrm{H}^{\text {Me-phen }}\right), 1.85(\mathrm{~m}, 2 \mathrm{H}$, $\left.\mathrm{H}^{\mathrm{NCH}_{2} \mathrm{CH}_{2}}\right), 1.15-1.05\left(\mathrm{~m}, 10 \mathrm{H}, \mathrm{H}^{\mathrm{CH}_{2}}\right), 0.68\left(\mathrm{~m}, 3 \mathrm{H}, \mathrm{H}^{\text {Me-octyl }}\right) .{ }^{13} \mathrm{C}$ NMR (126 MHz, DMSO- $\left.d_{6}\right) \delta /$ ppm: $156.2\left(\mathrm{C}^{\mathrm{B} 2 / \mathrm{B} 9}\right), 155.7\left(\mathrm{C}^{\mathrm{B} 2 / \mathrm{B} 9}\right)$, $152.4\left(\mathrm{C}^{\text {imid-2 }}\right), 139.7\left(\mathrm{C}^{\mathrm{C} 9 \mathrm{a}}\right), 137.8\left(\mathrm{C}^{\mathrm{A} 4}\right), 131.6\left(\mathrm{C}^{\mathrm{A} 2}\right), 130.0\left(\mathrm{C}^{\mathrm{B} 7}\right)$, $129.3\left(\mathrm{C}^{\mathrm{B} 4}\right), 129.2\left(\mathrm{C}^{\mathrm{A} 1}\right), 126.6\left(\mathrm{C}^{\mathrm{A} 3}\right), 126.2\left(\mathrm{C}^{\mathrm{C} 2}\right), 124.4\left(\mathrm{C}^{\mathrm{B} 5}\right)$, $123.5\left(\mathrm{C}^{\mathrm{B} 8}\right), 123.0\left(\mathrm{C}^{\mathrm{B} 3}\right), 122.8\left(\mathrm{C}^{\mathrm{C} 4 \mathrm{a}}\right), 121.4\left(\mathrm{C}^{\mathrm{B} 6 \mathrm{a}}\right), 120.5\left(\mathrm{C}^{\mathrm{C} 4}\right)$, $120.3\left(\mathrm{C}^{\mathrm{C} 3}\right), 117.4\left(\mathrm{C}^{\mathrm{B} 4 \mathrm{a}}\right), 109.5\left(\mathrm{C}^{\mathrm{C} 1}\right), 45.7\left(\mathrm{C}^{\mathrm{NCH}_{2}}\right), 30.9\left(\mathrm{C}^{\mathrm{CH}_{2}}\right)$, $28.9\left(\mathrm{C}^{\mathrm{NCH}_{2} \mathrm{CH}_{2}}\right), 28.0\left(\mathrm{C}^{\mathrm{CH}_{2}}\right), 25.0\left(\mathrm{C}^{\mathrm{CH}_{2}}\right), 24.6$ (overlapping $\left.\mathrm{C}^{\text {Me-phen }}\right), 21.7\left(\mathrm{C}^{\mathrm{CH}_{2}}\right), 18.4\left(\mathrm{C}^{\mathrm{CH}_{2}}\right), 13.6\left(\mathrm{C}^{\mathrm{Me}-o c t y l}\right),\left(\mathrm{C}^{\mathrm{B} 6}, \mathrm{C}^{\mathrm{B} 10 \mathrm{a}}\right.$, $\mathrm{C}^{\mathrm{B} 10 \mathrm{~b}}$ not resolved). UV-Vis $\left(\mathrm{CH}_{2} \mathrm{Cl}_{2}, 1.00 \times 10^{-5} \mathrm{~mol} \mathrm{dm}^{-3}\right)$ $\lambda / \mathrm{nm}\left(\varepsilon / \mathrm{dm}^{3} \mathrm{~mol}^{-1} \mathrm{~cm}^{-1}\right) 294$ (55 000), $310 \mathrm{sh}$ (35 500), $325 \mathrm{sh}$ (29 900), 340 (23 000), $370 \mathrm{sh}$ (4200). ESI MS m/z: $602.6[\mathrm{M}+\mathrm{H}]^{+}$ (calc. 602.3). Found: $\mathrm{C} 78.95, \mathrm{H} 6.56, \mathrm{~N} 10.80 ; \mathrm{C}_{41} \mathrm{H}_{39} \mathrm{~N}_{5} \cdot \mathrm{H}_{2} \mathrm{O}$ requires $\mathrm{C} 79.45, \mathrm{H} 6.67, \mathrm{~N} 11.30 \%$.

Compound $5 \mathrm{~K}_{3} \mathrm{PO}_{4}(343 \mathrm{mg}, 1.62 \mathrm{mmol})$ was dissolved in $\mathrm{H}_{2} \mathrm{O}(2 \mathrm{ml})$ under $\mathrm{N}_{2}$, and $\mathrm{N}_{2}$ was bubbled through the solution for $10 \mathrm{~min}$. In a separate flask, $\mathrm{N}_{2}$ was bubbled through 1,4-dioxane $(5 \mathrm{ml})$ for $10 \mathrm{~min}$ and then 4,7-dichloro-2,9dimethyl-1,10-phenanthroline $(100 \mathrm{mg}, 0.361 \mathrm{mmol})$, 4-(diphenylamino)phenylboronic acid $(230 \mathrm{mg}, 0.794 \mathrm{mmol})$ and catalytic amounts of $\left[\mathrm{Pd}(\mathrm{dba})_{3}\right](24.7 \mathrm{mg}, 0.0238 \mathrm{mmol}, 6.6 \mathrm{~mol} \%)$ and tricyclohexylphosphine $(15.2 \mathrm{mg}, 0.0542 \mathrm{mmol})$ were added. The aqueous solution of $\mathrm{K}_{3} \mathrm{PO}_{4}$ was then added to the reaction mixture and this was heated at reflux at $95{ }^{\circ} \mathrm{C}$ for $15 \mathrm{~h}$. The orange mixture was allowed to cool down to room temperature and water $(10 \mathrm{ml})$ was added. The mixture was extracted with $\mathrm{CH}_{2} \mathrm{Cl}_{2}(5 \times 15 \mathrm{ml})$ and the organic layer was dried over $\mathrm{Na}_{2} \mathrm{SO}_{4}$ and the solvent then removed. The product was recrystallized from $\mathrm{CH}_{2} \mathrm{Cl}_{2} / \mathrm{MeOH}(1: 1)$ and 5 was isolated as a yellow solid (97.0 mg, $0.140 \mathrm{mmol}, 38.7 \%$ ). ${ }^{1} \mathrm{H}$ NMR 
$\left(500 \mathrm{MHz}, \mathrm{CDCl}_{3}\right) \delta / \mathrm{ppm} 8.00\left(\mathrm{~s}, 2 \mathrm{H}, \mathrm{H}^{\mathrm{B} 5}\right), 7.55\left(\mathrm{~s}, 2 \mathrm{H}, \mathrm{H}^{\mathrm{B} 3}\right), 7.41$ $\left(\mathrm{m}, 4 \mathrm{H}, \mathrm{H}^{\mathrm{C} 2}\right), 7.32\left(\mathrm{~m}, 8 \mathrm{H}, \mathrm{H}^{\mathrm{A3}}\right), 7.19\left(\mathrm{~m}, 12 \mathrm{H}, \mathrm{H}^{\mathrm{A} 2+\mathrm{C} 3}\right), 7.10(\mathrm{~m}$, $\left.4 \mathrm{H}, \mathrm{H}^{\mathrm{A} 4}\right), 3.14\left(\mathrm{~s}, 6 \mathrm{H}, \mathrm{H}^{\mathrm{Me}}\right) .{ }^{13} \mathrm{C}\left(126 \mathrm{MHz}, \mathrm{CDCl}_{3}\right) \delta / \mathrm{ppm} 158.8$ $\left(\mathrm{C}^{\mathrm{B} 2}\right), 150.2\left(\mathrm{C}^{\mathrm{B} 4}\right), 148.9\left(\mathrm{C}^{\mathrm{C} 4}\right), 147.3\left(\mathrm{C}^{\mathrm{A} 1}\right), 142.9\left(\mathrm{C}^{\mathrm{B} 10 \mathrm{a}}\right), 130.8$ $\left(\mathrm{C}^{\mathrm{C} 2}\right), 129.7\left(\mathrm{C}^{\mathrm{A} 3}\right), 125.2\left(\mathrm{C}^{\mathrm{A} 2}\right), 125.1\left(\mathrm{C}^{\mathrm{B} 4 \mathrm{a}}\right), 124.8\left(\mathrm{C}^{\mathrm{B} 3}\right), 123.9\left(\mathrm{C}^{\mathrm{A} 4}\right)$, $123.5\left(\mathrm{C}^{\mathrm{B} 5}\right), 122.5\left(\mathrm{C}^{\mathrm{C} 3}\right), 24.7\left(\mathrm{C}^{\mathrm{Me}}\right),\left(\mathrm{C}^{\mathrm{C} 1}\right.$ not resolved $)$. UV-Vis $\left(\mathrm{CH}_{2} \mathrm{Cl}_{2}, 1.00 \times 10^{-5} \mathrm{~mol} \mathrm{dm}^{-3}\right) \lambda / \mathrm{nm}\left(\varepsilon / \mathrm{dm}^{3} \mathrm{~mol}^{-1} \mathrm{~cm}^{-1}\right) 297$ (49 000), $360 \mathrm{sh}$ (18 100), $500 \mathrm{sh}$ (4950). HR ESI-MS m/z: 695.3174 $[\mathrm{M}+\mathrm{H}]^{+}$(calc. 695.3169). Satisfactory elemental analysis was not obtained.

$\left[\mathbf{C u}(\mathbf{1})_{2}\right]\left[\mathrm{PF}_{6}\right]$. A solution of $\left[\mathrm{Cu}(\mathrm{NCMe})_{4}\right]\left[\mathrm{PF}_{6}\right](16.2 \mathrm{mg}$, $0.0435 \mathrm{mmol})$ in $\mathrm{MeCN}(2 \mathrm{ml})$ was added dropwise to a solution of $1(44.8 \mathrm{mg}, 0.0870 \mathrm{mmol})$ in $\mathrm{CHCl}_{3}(1 \mathrm{ml})$. The dark red solution was stirred for $3 \mathrm{~h}$ and then the solvent was removed. The product was purified by column chromatography (alumina, $\mathrm{CH}_{2} \mathrm{Cl}_{2}$ with $\left.1 \% \mathrm{MeOH}\right)$ and $\left[\mathrm{Cu}(\mathbf{1})_{2}\right]\left[\mathrm{PF}_{6}\right]$ was isolated as a dark red solid (38.0 mg, $0.0310 \mathrm{mmol}, 70.5 \%) .{ }^{1} \mathrm{H}$ NMR $(500 \mathrm{MHz}$, $\left.\mathrm{CD}_{3} \mathrm{CN}\right) \delta / \mathrm{ppm} 9.10\left(\mathrm{~d}, J=8.2 \mathrm{~Hz}, 2 \mathrm{H}, \mathrm{H}^{\mathrm{B} 7}\right), 8.91(\mathrm{~d}, J=8.7 \mathrm{~Hz}$, $\left.2 \mathrm{H}, \mathrm{H}^{\mathrm{B} 4}\right), 7.91\left(\mathrm{~d}, J=8.6 \mathrm{~Hz}, 2 \mathrm{H}, \mathrm{H}^{\mathrm{B} 3}\right), 7.89(\mathrm{~d}, J=8.3 \mathrm{~Hz}, 2 \mathrm{H}$, $\left.\mathrm{H}^{\mathrm{B} 8}\right), 7.82\left(\mathrm{~m}, 4 \mathrm{H}, \mathrm{H}^{\mathrm{A} 3}\right), 7.73\left(\mathrm{~m} 4 \mathrm{H}, \mathrm{H}^{\mathrm{A} 2}\right), 4.73(\mathrm{t}, J=7.3 \mathrm{~Hz}, 4 \mathrm{H}$, $\left.\mathrm{H}^{\mathrm{NCH}_{2}}\right), 2.44\left(\mathrm{~s}, 6 \mathrm{H}, \mathrm{H}^{\mathrm{Me}-\mathrm{phen}}\right), 2.43\left(\mathrm{~s}, 6 \mathrm{H}, \mathrm{H}^{\mathrm{Me}-\mathrm{phen}}\right), 1.91(\mathrm{~m}, 4 \mathrm{H}$, $\left.\mathrm{H}^{\mathrm{CH}_{2}}\right), 1.24-1.18\left(\mathrm{~m}, 8 \mathrm{H}, \mathrm{H}^{\mathrm{CH}_{2}}\right), 1.16-1.11\left(\mathrm{~m}, 12 \mathrm{H}, \mathrm{H}^{\mathrm{CH}_{2}}\right), 0.83$ $\left(\mathrm{t}, J=7.2 \mathrm{~Hz}, 6 \mathrm{H}, \mathrm{H}^{\mathrm{Me}-\mathrm{octyl}}\right) \cdot{ }^{13} \mathrm{C} \mathrm{NMR}\left(126 \mathrm{MHz}, \mathrm{CD}_{3} \mathrm{CN}\right) \delta / \mathrm{ppm}$ $157.1\left(\mathrm{C}^{\mathrm{B} 2 / \mathrm{B} 9}\right), 156.6\left(\mathrm{C}^{\mathrm{B} 2 / \mathrm{B} 9}\right), 154.6\left(\mathrm{C}^{\mathrm{imid}-2}\right), 142.5\left(\mathrm{C}^{\mathrm{B} 10 \mathrm{a} / \mathrm{B} 10 \mathrm{~b}}\right)$, $142.0\left(\mathrm{C}^{\mathrm{B} 10 \mathrm{a} / \mathrm{B} 10 \mathrm{~b}}\right), 137.1\left(\mathrm{C}^{\mathrm{B} 4 \mathrm{a} / \mathrm{B} 6 \mathrm{a}}\right), 133.1\left(\mathrm{C}^{\mathrm{A} 3}\right), 132.8\left(\mathrm{C}^{\mathrm{A} 2}\right), 132.1$ $\left(\mathrm{C}^{\mathrm{B} 7}\right), 131.4\left(\mathrm{C}^{\mathrm{B} 4}\right), 130.6\left(\mathrm{C}^{\mathrm{A} 1}\right), 126.9\left(\mathrm{C}^{\mathrm{B} 8}\right), 126.4\left(\mathrm{C}^{\mathrm{B}}\right), 125.0$ $\left(\mathrm{C}^{\mathrm{A} 4}\right), 124.8\left(\mathrm{C}^{\mathrm{B} 4 \mathrm{a} / \mathrm{B6a}}\right), 123.9\left(\mathrm{C}^{\mathrm{B} 6}\right), 120.0\left(\mathrm{C}^{\mathrm{B} 5}\right), 47.5\left(\mathrm{C}^{\mathrm{NCH}_{2}}\right), 32.2$ $\left(\mathrm{C}^{\mathrm{CH}_{2}}\right), 29.4\left(2 \mathrm{C}^{\mathrm{CH}_{2}}\right), 26.6\left(\mathrm{C}^{\mathrm{CH}_{2}}\right), 25.8\left(2 \mathrm{C}^{\mathrm{Me}-\mathrm{phen}}\right), 23.3\left(\mathrm{C}^{\mathrm{CH}_{2}}\right)$, $14.3\left(\mathrm{C}^{\mathrm{Me}-\text { octyl }}\right)$. UV-Vis $\left(\mathrm{CH}_{2} \mathrm{Cl}_{2}, 1.00 \times 10^{-5} \mathrm{~mol} \mathrm{dm}{ }^{-3}\right) \lambda / \mathrm{nm}$ $\left(\varepsilon / \mathrm{dm}^{3} \mathrm{~mol}^{-1} \mathrm{~cm}^{-1}\right) 282$ (87 200), 304 (95 300), 474 (13 400). ESI MS $m / z: 1093.7\left[\mathrm{M}-\mathrm{PF}_{6}\right]^{+}$(calc. 1093.3). Found C 57.02, H 5.38, $\mathrm{N}$ 9.01; $\mathrm{C}_{58} \mathrm{H}_{62} \mathrm{Br}_{2} \mathrm{CuF}_{6} \mathrm{~N}_{8} \mathrm{P}$ requires C 56.20, H 5.04, $\mathrm{N}$ 9.04\%.

$\left[\mathbf{C u}(3)_{2}\right]\left[\mathbf{P F}_{6}\right]$. The method and purification were as for $\left[\mathrm{Cu}(\mathbf{1})_{2}\right]\left[\mathrm{PF}_{6}\right]$; reagents and solvents were $\left[\mathrm{Cu}(\mathrm{MeCN})_{4}\right]\left[\mathrm{PF}_{6}\right]$ $(12.5 \mathrm{mg}, 0.0334 \mathrm{mmol})$ in $\mathrm{MeCN}(2 \mathrm{ml})$ and $3(50 \mathrm{mg}, 0.0668$ $\mathrm{mmol})$ in $\mathrm{CHCl}_{3}(2 \mathrm{ml})$. $\left[\mathrm{Cu}(3)_{2}\right]\left[\mathrm{PF}_{6}\right]$ was isolated as a red solid (41.4 mg, $24.3 \mu \mathrm{mol}, 72.7 \%) .{ }^{1} \mathrm{H}$ NMR $\left(500 \mathrm{MHz}, \mathrm{CDCl}_{3}\right) \delta / \mathrm{ppm}$ $9.24\left(\mathrm{br}, 2 \mathrm{H}, \mathrm{H}^{\mathrm{B} 7}\right), 8.85\left(\mathrm{~d}, J=8.6 \mathrm{~Hz}, 2 \mathrm{H}, \mathrm{H}^{\mathrm{B} 4}\right), 7.94(\mathrm{~d}, J=$ $\left.8.5 \mathrm{~Hz}, 2 \mathrm{H}, \mathrm{H}^{\mathrm{B} 3}\right), 7.78\left(\mathrm{~d}, J=8.3 \mathrm{~Hz}, 2 \mathrm{H}, \mathrm{H}^{\mathrm{B} 8}\right), 7.54\left(\mathrm{~m}, 4 \mathrm{H}, \mathrm{H}^{\mathrm{A} 2}\right)$, $7.15\left(\mathrm{~m}, 8 \mathrm{H}, \mathrm{H}^{\mathrm{C} 2}\right), 7.07\left(\mathrm{~m}, 4 \mathrm{H}, \mathrm{H}^{\mathrm{A} 3}\right), 6.89\left(\mathrm{~m}, 8 \mathrm{H}, \mathrm{H}^{\mathrm{C} 3}\right), 4.78(\mathrm{t}$, $\left.J=6.8 \mathrm{~Hz}, 4 \mathrm{H}, \mathrm{H}^{\mathrm{NCH}_{2}}\right), 3.97\left(\mathrm{t}, J=6.4 \mathrm{~Hz}, 8 \mathrm{H}, \mathrm{H}^{\mathrm{OCH}_{2}}\right), 2.45(\mathrm{~s}$, $\left.6 \mathrm{H}, \mathrm{H}^{\text {Me-phen }}\right), 2.43\left(\mathrm{~s}, 6 \mathrm{H}, \mathrm{H}^{\text {Me-phen }}\right), 2.00\left(\mathrm{~m}, 4 \mathrm{H}, \mathrm{H}^{\mathrm{NCH}_{2} \mathrm{CH}_{2}}\right)$,

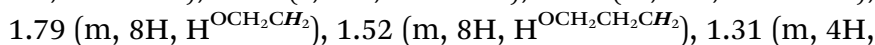

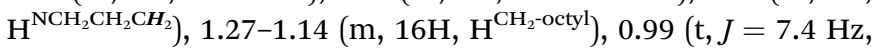
$\left.12 \mathrm{H}, \mathrm{H}^{\text {Me-butyl }}\right), 0.84\left(\mathrm{t}, J=6.8 \mathrm{~Hz}, 6 \mathrm{H}, \mathrm{H}^{\text {Me-octyl }}\right) .{ }^{13} \mathrm{C}$ NMR $\left(126 \mathrm{MHz}, \mathrm{CDCl}_{3}\right) \delta / \mathrm{ppm} 156.1\left(\mathrm{C}^{\mathrm{C} 4}\right), 155.3\left(\mathrm{C}^{\mathrm{B} 2 / \mathrm{B} 9}\right), 155.2$ $\left(\mathrm{C}^{\text {imid-2}}\right), 154.8\left(\mathrm{C}^{\mathrm{B} 2 / \mathrm{B} 9}\right), 150.4\left(\mathrm{C}^{\mathrm{A} 4}\right), 141.1\left(\mathrm{C}^{\mathrm{B} 10 \mathrm{~b}}\right), 139.6\left(\mathrm{C}^{\mathrm{C} 1}\right)$, $131.7\left(\mathrm{C}^{\mathrm{B} 7}\right), 130.5\left(\mathrm{C}^{\mathrm{A} 2}\right), 129.9\left(\mathrm{C}^{\mathrm{B} 4}\right), 127.4\left(\mathrm{C}^{\mathrm{C} 2}\right), 125.6\left(\mathrm{C}^{\mathrm{B} 3}\right)$, 125.3 $\left(\mathrm{C}^{\mathrm{B} 8}\right), 124.8\left(\mathrm{C}^{\mathrm{B} 5}\right), 122.8\left(\mathrm{C}^{\mathrm{B} 6 \mathrm{a}}\right), 120.0\left(\mathrm{C}^{\mathrm{A} 1}\right), 119.2\left(\mathrm{C}^{\mathrm{B} 4 \mathrm{a}}\right)$, $118.8\left(\mathrm{C}^{\mathrm{A} 3}\right), 115.6\left(\mathrm{C}^{\mathrm{C} 3}\right), 67.8\left(\mathrm{C}^{\mathrm{OCH}_{2}}\right), 46.7\left(\mathrm{C}^{\mathrm{NCH}_{2}}\right), 31.6$ $\left(\mathrm{C}^{\mathrm{CH}_{2} \text {-octyl }}\right), 31.2\left(\mathrm{C}^{\mathrm{OCH}_{2} \mathrm{CH}_{2}}\right), 30.0\left(\mathrm{C}^{\mathrm{NCH}_{2} \mathrm{CH}_{2}}\right), 29.0\left(\mathrm{C}^{\mathrm{CH}_{2} \text {-octyl }}\right), 28.9$ $\left(\mathrm{C}^{\mathrm{CH}_{2} \text {-octyl }}\right), 26.0\left(\mathrm{C}^{\mathrm{CH}_{2} \text {-octyl }}\right), 25.9\left(\mathrm{C}^{\mathrm{Me}-\text { phen }}\right), 25.7\left(\mathrm{C}^{\mathrm{Me}-\mathrm{phen}}\right), 22.4$ $\left(\mathrm{C}^{\mathrm{CH}_{2} \text {-octyl }}\right), 18.9\left(\mathrm{C}^{\mathrm{OCH}_{2} \mathrm{CH}_{2} \mathrm{CH}_{2}}\right), 13.9\left(\mathrm{C}^{\mathrm{Me} \text {-octyl }}\right), 13.7\left(\mathrm{C}^{\text {Me-butyl }}\right)$, $\left(\mathrm{C}^{\mathrm{B} 6}, \mathrm{C}^{\mathrm{B} 10 \mathrm{a}}\right.$ not resolved $)$. UV-Vis $\left(\mathrm{CH}_{2} \mathrm{Cl}_{2}, 1.00 \times 10^{-5}\right.$ mol dm $\left.{ }^{-3}\right) \lambda / \mathrm{nm}\left(\varepsilon / \mathrm{dm}^{3} \mathrm{~mol}^{-1} \mathrm{~cm}^{-1}\right) 256$ (73 200), 292 (88 800), 342 sh $(58700), 469$ (16 100). HR ESI-MS $m / z: 1557.8334$
$\left[\mathrm{M}-\mathrm{PF}_{6}\right]^{+}$(calc. 1557.8315). Satisfactory elemental analysis could not be obtained.

$\left[\mathbf{C u}(4)_{2}\right]\left[\mathbf{P F}_{6}\right]$. The method and purification were as for $\left[\mathrm{Cu}(\mathbf{1})_{2}\right]\left[\mathrm{PF}_{6}\right]$; reagents and solvents were $\left[\mathrm{Cu}(\mathrm{NCMe})_{4}\right]\left[\mathrm{PF}_{6}\right]$ $(12.4 \mathrm{mg}, 0.0332 \mathrm{mmol})$ in $\mathrm{MeCN}(5 \mathrm{~mL})$ and $4(40.0 \mathrm{mg}$, $0.0665 \mathrm{mmol})$ in $\mathrm{CH}_{2} \mathrm{Cl}_{2}(5 \mathrm{ml})$. $\left[\mathrm{Cu}(4)_{2}\right]\left[\mathrm{PF}_{6}\right]$ was isolated as a dark red solid (46.9 mg, $0.0330 \mathrm{mmol}, 100 \%) .{ }^{1} \mathrm{H}$ NMR $\left(500 \mathrm{MHz}, \mathrm{CD}_{3} \mathrm{CN}\right) \delta / \mathrm{ppm}: 9.18\left(\mathrm{~d}, J=8.2 \mathrm{~Hz}, 2 \mathrm{H}, \mathrm{H}^{\mathrm{B} 7}\right), 8.99(\mathrm{~d}$, $\left.J=8.6 \mathrm{~Hz}, 2 \mathrm{H}, \mathrm{H}^{\mathrm{B} 4}\right), 8.27\left(\mathrm{dt}, J=7.8,1.0 \mathrm{~Hz}, 4 \mathrm{H}, \mathrm{H}^{\mathrm{C} 4}\right), 8.11(\mathrm{~m}$, $\left.4 \mathrm{H}, \mathrm{H}^{\mathrm{A} 2 / \mathrm{A} 3}\right), 7.97\left(\mathrm{~d}, J=8.5 \mathrm{~Hz}, 2 \mathrm{H}, \mathrm{H}^{\mathrm{B} 3}\right), 7.94$ (overlapping $\mathrm{m}$, $\left.6 \mathrm{H}, \mathrm{H}^{\mathrm{A} 2 / \mathrm{A} 3+\mathrm{B} 8}\right), 7.64\left(\mathrm{~m}, 4 \mathrm{H}, \mathrm{H}^{\mathrm{C} 1}\right), 7.51(\mathrm{ddd}, J=8.3,7.0,1.2 \mathrm{~Hz}$, $\left.4 \mathrm{H}, \mathrm{H}^{\mathrm{C} 2}\right), 7.36\left(\mathrm{ddd}, J=8.0,7.0,0.9 \mathrm{~Hz}, 4 \mathrm{H}, \mathrm{H}^{\mathrm{C} 3}\right), 4.90(\mathrm{t}, J=$ $\left.7.2 \mathrm{~Hz}, 4 \mathrm{H}, \mathrm{H}^{\mathrm{NCH}_{2}}\right), 2.505$ (s, 6H, He-Phen $), 2.50\left(\mathrm{~s}, 6 \mathrm{H}, \mathrm{H}^{\mathrm{Me}-\mathrm{Phen}}\right)$,

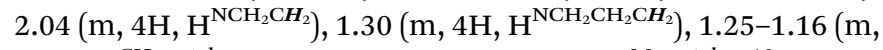
$\left.16 \mathrm{H}, \mathrm{H}^{\mathrm{CH}_{2} \text {-octyl }}\right), 0.76\left(\mathrm{t}, J=6.9 \mathrm{~Hz}, 6 \mathrm{H}, \mathrm{H}^{\mathrm{Me} \text {-octyl }}\right) .{ }^{13} \mathrm{C} \mathrm{NMR}$ $\left(126 \mathrm{MHz}, \mathrm{CD}_{3} \mathrm{CN}\right) \delta / \mathrm{ppm} 156.5\left(\mathrm{C}^{\mathrm{B} 2 / \mathrm{B} 9}\right), 155.5\left(\mathrm{C}^{\mathrm{B} 2 / \mathrm{B} 9}\right), 154.2$ $\left(\mathrm{C}^{\mathrm{imid}-2}\right), 141.5\left(\mathrm{C}^{\mathrm{B} 10 \mathrm{~b}}\right), 141.1\left(\mathrm{C}^{\mathrm{B} 10 \mathrm{a}}\right), 140.6\left(\mathrm{C}^{\mathrm{C} 9 \mathrm{a}}\right), 139.0\left(\mathrm{C}^{\mathrm{A} 4}\right)$, $136.2\left(\mathrm{C}^{\mathrm{B} 6}\right), 131.7\left(\mathrm{C}^{\mathrm{A} 2}\right), 131.2\left(\mathrm{C}^{\mathrm{B} 7}\right), 130.6\left(\mathrm{C}^{\mathrm{B} 4}\right), 129.5\left(\mathrm{C}^{\mathrm{A} 1}\right)$, $127.3\left(\mathrm{C}^{\mathrm{A} 3}\right), 126.3\left(\mathrm{C}^{\mathrm{C} 2}\right), 126.0\left(\mathrm{C}^{\mathrm{B} 8}\right), 125.6\left(\mathrm{C}^{\mathrm{B} 3}\right), 125.3\left(\mathrm{C}^{\mathrm{B} 5}\right)$, $123.5\left(\mathrm{C}^{\mathrm{C} 4 \mathrm{a}}\right), 123.1\left(\mathrm{C}^{\mathrm{B} 6 \mathrm{a}}\right), 120.5\left(\mathrm{C}^{\mathrm{C} 4}\right), 120.3\left(\mathrm{C}^{\mathrm{C} 3}\right), 119.2\left(\mathrm{C}^{\mathrm{B} 4 \mathrm{a}}\right)$, $109.8\left(\mathrm{C}^{\mathrm{Cl}}\right), 46.6\left(\mathrm{C}^{\mathrm{NCH}_{2}}\right), 31.3\left(\mathrm{C}^{\mathrm{CH}_{2}-\mathrm{octyl}}\right), 29.6\left(\mathrm{C}^{\mathrm{NCH}_{2} \mathrm{CH}_{2}}\right), 28.5$ $\left(2 \mathrm{C}^{\mathrm{CH}_{2} \text {-octyl }}\right), 25.6\left(\mathrm{C}^{\mathrm{NCH}_{2} \mathrm{CH}_{2} \mathrm{CH}_{2}}\right), 25.0$ (overlapping $\left.\mathrm{C}^{\mathrm{Me}-\mathrm{Phen}}\right), 22.3$ $\left(\mathrm{C}^{\mathrm{CH}_{2} \text {-octyl }}\right), 13.3\left(\mathrm{C}^{\mathrm{Me} \text {-octyl }}\right)$. UV-Vis $\left(\mathrm{CH}_{2} \mathrm{Cl}_{2}, 1.00 \times 10^{-5}\right.$ mol dm $\left.{ }^{-3}\right) \lambda / \mathrm{nm}\left(\varepsilon / \mathrm{dm}^{3} \mathrm{~mol}^{-1} \mathrm{~cm}^{-1}\right) 262$ (106 400), 292 (104 300), 338 (52 900), 475 (14 500). ESI MS $m / z: 1266.0$ $\left[\mathrm{M}-\mathrm{PF}_{6}\right]^{+}$(calc. 1265.6). HR ESI-MS: $m / z$ 1265.5687 [M - $\left.\mathrm{PF}_{6}\right]^{+}$ (calc. 1265.5701). Found C 69.00, H 5.78, N 9.74; $\mathrm{C}_{82} \mathrm{H}_{78} \mathrm{CuF}_{6}-$ $\mathrm{N}_{10} \mathrm{P} \cdot 0.5 \mathrm{H}_{2} \mathrm{O}$ requires C 69.30, H 5.60, $\mathrm{N} 9.86 \%$.

$\left[\mathbf{C u}(5)_{2}\right]\left[\mathbf{P F}_{6}\right]$. A solution of $\left[\mathrm{Cu}(\mathrm{NCMe})_{4}\right]\left[\mathrm{PF}_{6}\right](22.8 \mathrm{mg}$, $0.0612 \mathrm{mmol})$ in MeCN $(2 \mathrm{~mL})$ was added dropwise to a solution of $28(85.0 \mathrm{mg}, 0.122 \mathrm{mmol})$ in $\mathrm{CHCl}_{3}(2 \mathrm{~mL})$. The dark red solution was stirred for $0.5 \mathrm{~h}$ and the solvent was then removed. The residue was suspended in water and the mixture then filtered. The filter cake was collected by dissolving it in a mixture of $\mathrm{MeCN}$ and $\mathrm{CHCl}_{3}$ and then filtering the solution through a P3 glass filter $(16-40 \mu \mathrm{m})$. After removing the solvent from the filtrate in vacuo, $\left[\mathrm{Cu}(5)_{2}\right]\left[\mathrm{PF}_{6}\right]$ was isolated as a red solid $(91.0 \mathrm{mg}$, $0.057 \mathrm{mmol}, 93.0 \%) .{ }^{1} \mathrm{H}$ NMR $\left(500 \mathrm{MHz}, \mathrm{CD}_{2} \mathrm{Cl}_{2}\right) \delta / \mathrm{ppm} 8.21(\mathrm{br}$, $\left.4 \mathrm{H}, \mathrm{H}^{\mathrm{B} 5}\right), 7.72\left(\mathrm{br}, 4 \mathrm{H}, \mathrm{H}^{\mathrm{B} 3}\right), 7.51\left(\mathrm{~m}, 8 \mathrm{H}, \mathrm{H}^{\mathrm{C} 2}\right), 7.35(\mathrm{~m}, 16 \mathrm{H}$, $\left.\mathrm{H}^{\mathrm{A} 3}\right), 7.23\left(\mathrm{~m}, 24 \mathrm{H}, \mathrm{H}^{\mathrm{A} 2+\mathrm{C} 3}\right), 7.14\left(\mathrm{~m}, 8 \mathrm{H}, \mathrm{H}^{\mathrm{A} 4}\right), 2.53(\mathrm{~s}, 12 \mathrm{H}$, $\left.\mathrm{H}^{\text {Me-phen }}\right) .{ }^{13} \mathrm{C}$ NMR $\left(126 \mathrm{MHz}, \mathrm{CD}_{2} \mathrm{Cl}_{2}\right) \delta / \mathrm{ppm} 157.2\left(\mathrm{C}^{\mathrm{B} 2}\right), 149.6$ $\left(\mathrm{C}^{\mathrm{B} 4}\right), 149.3\left(\mathrm{C}^{\mathrm{C} 4}\right), 147.5\left(\mathrm{C}^{\mathrm{A} 1}\right), 144.5\left(\mathrm{C}^{\mathrm{B} 10 \mathrm{a}}\right), 131.0\left(\mathrm{C}^{\mathrm{C} 2}\right), 130.0$ $\left(\mathrm{C}^{\mathrm{A} 3}\right), 126.0\left(\mathrm{C}^{\mathrm{B} 4 \mathrm{a}}\right), 125.8\left(\mathrm{C}^{\mathrm{C} 3 / \mathrm{A} 2}\right), 125.7\left(\mathrm{C}^{\mathrm{B} 3}\right), 124.4\left(\mathrm{C}^{\mathrm{A} 4}\right), 122.5$ $\left(\mathrm{C}^{\mathrm{C} 3 / \mathrm{A} 2}\right), 124.1\left(\mathrm{C}^{\mathrm{B} 5}\right), 26.2\left(\mathrm{C}^{\mathrm{Me}-\mathrm{phen}}\right),\left(\mathrm{C}^{\mathrm{C} 1}\right.$ not resolved $)$. UV-Vis $\left(\mathrm{CH}_{2} \mathrm{Cl}_{2}, 1.00 \times 10^{-5} \mathrm{~mol} \mathrm{dm}{ }^{-3}\right) \lambda / \mathrm{nm}\left(\varepsilon / \mathrm{dm}^{3} \mathrm{~mol}^{-1} \mathrm{~cm}^{-1}\right) 295$ (119 200), 384 (54 000), 486 (23 600). ESI MS $\mathrm{m} / \mathrm{z}: 1452.1$ $\left[\mathrm{M}-\mathrm{PF}_{6}\right]^{+}$(calc. 1452.6). Found $\mathrm{C}$ 72.47, $\mathrm{H}$ 4.79, $\mathrm{N}$ 7.01; $\mathrm{C}_{100} \mathrm{H}_{76} \mathrm{CuF}_{6} \mathrm{~N}_{8} \mathrm{P} \cdot 3 \mathrm{H}_{2} \mathrm{O}$ requires $\mathrm{C} 72.69, \mathrm{H} 5.00, \mathrm{~N} 6.78 \%$.

\section{Crystallography}

Single crystal data were collected on a Bruker APEX-II diffractometer with data reduction, solution and refinement using the programs APEX $^{34}$ and CRYSTALS. ${ }^{35}$ Structure analysis used Mercury v. 3.5.1. ${ }^{36,37}$

Compound $4 \mathrm{C}_{41} \mathrm{H}_{39} \mathrm{~N}_{5}, M=601.79$, colourless needle, triclinic, space group $P \overline{1}, a=11.7635(14), b=11.8126(14), c=$ 
12.2155(14) $\mathrm{A}, \alpha=72.343(4), \beta=86.285(4), \gamma=83.168(4)^{\circ}, U=$ 1605.25(18) $\AA^{3}, Z=2, D_{\mathrm{c}}=1.245 \mathrm{Mg} \mathrm{m}^{-3}, \mu(\mathrm{Cu}-\mathrm{K} \alpha)=$ $0.570 \mathrm{~mm}^{-1}, T=123 \mathrm{~K}$. Total 18881 reflections, 5769 unique, $R_{\text {int }}=0.027$. Refinement of 5522 reflections (415 parameters) with $I>2 \sigma(I)$ converged at final $R 1=0.0375$ ( $R 1$ all data $=$ $0.0391), \mathrm{w} R 2=0.0416(\mathrm{w} R 2$ all data $=0.0477)$, gof $=1.1112$. CCDC 1405837. $\dagger$

\section{DSC fabrication and measurements}

Solaronix Test Cell Titania Electrodes were used for the photoanodes. They were washed with milliQ $\mathrm{H}_{2} \mathrm{O}$ and heated at $450^{\circ} \mathrm{C}$ for $30 \mathrm{~min}$, then cooled to $\approx 80{ }^{\circ} \mathrm{C}$ and soaked in a $1.0 \mathrm{mM}$ DMSO solution of 6 for $24 \mathrm{~h}$ at room temperature. After removal of the electrodes from the solution, they were washed with DMSO and EtOH and dried in a stream of $\mathrm{N}_{2}$. Each functionalized electrode was then soaked for 3 days in a $0.1 \mathrm{mM}$ MeCN solution of $\left[\mathrm{Cu}\left(\mathrm{L}_{\text {ancillary }}\right)_{2}\right]\left[\mathrm{PF}_{6}\right]\left(\mathrm{L}_{\text {ancillary }}=1-5\right)$ at room temperature. The electrodes were removed from the dye-bath, washed with MeCN and dried in a stream of $\mathrm{N}_{2}$.

N719 reference electrodes were made by dipping Solaronix Test Cell Titania Electrodes in a $0.3 \mathrm{mM}$ EtOH solution of N719 (Solaronix) for 3 days. The electrodes were removed from the dyebath, and were washed with EtOH and dried in a stream of $\mathrm{N}_{2}$.

For the counter electrodes, Solaronix Test Cell Platinum Electrodes were used, and volatile organic impurities were removed by heating on a heating plate at $450{ }^{\circ} \mathrm{C}$ for $30 \mathrm{~min}$.

The dye-covered $\mathrm{TiO}_{2}$ electrode and $\mathrm{Pt}$ counter-electrode were combined using thermoplast hot-melt sealing foil (Solaronix Test Cell Gaskets, $60 \mu \mathrm{m}$ ) by heating while pressing them together. The electrolyte ( $\mathrm{LiI}(0.1 \mathrm{M}), \mathrm{I}_{2}(0.05 \mathrm{M})$, 1-methylbenzimidazole $(0.5 \mathrm{M})$, 1-butyl-3-methylimidazolinium iodide $(0.6 \mathrm{M})$ in 3-methoxypropionitrile) was introduced into the DSC by vacuum backfilling. The hole in the counter electrode was sealed with hot-melt sealing foil (Solaronix Test Cell Sealings) and a cover glass (Solaronix Test Cell Caps).

For each dye, duplicate DSCs were made and the cells were completely masked. ${ }^{38,39}$ Measurements were made by irradiating the DSC from behind using a SolarSim 150 (Solaronix) light source previously calibrated with a silicon reference cell to $100 \mathrm{~mW} \mathrm{~cm}^{-2}$ (1 sun).

External quantum efficiency (EQE) measurements were made using a Spe-Quest quantum efficiency instrument from Rera Systems (Netherlands) with a $100 \mathrm{~W}$ halogen lamp (QTH) and a lambda 300 grating monochromator (Lot Oriel). The monochromatic light was modulated to $3 \mathrm{~Hz}$ using a chopper wheel (ThorLabs). The cell response was amplified with a large dynamic range IV converter (CVI Melles Griot) and measured with a SR830 DSP Lock-In amplifier (Stanford Research).

\section{Results and discussion}

\section{Synthesis and characterization of ancillary ligands 1-5}

We have previously reported the preparation and characterization of the bromo-derivative $\mathbf{1}$ and subsequent Hartwig-Buchwald amination with diphenylamine to give 2 (Scheme 2). ${ }^{26}$ The same strategy was used for the synthesis of 3 . 4,4'-Di- $n$ - butoxydiphenylamine was prepared by a copper(I) iodide/L-proline catalysed $^{32}$ Ullmann coupling (Scheme 3a) and was then used in the Hartwig-Buchwald amination shown in scheme $3 \mathrm{~b}$. Compound 3 was isolated in $51.2 \%$ yield. Attempts to prepare 4 by reaction of 1 with $9 H$-carbazole using Hartwig-Buchwald conditions led to very low yields of $\mathbf{4}$; changing the catalyst from $\left[\mathrm{Pd}(\mathrm{dba})_{2}\right] / \mathrm{P}^{t} \mathrm{Bu}_{3}$ to $\left[\mathrm{Pd}(\mathrm{OAc})_{2}\right] / \mathrm{P}^{t} \mathrm{Bu}_{3}$ or $\left[\mathrm{Pd}\left(\mathrm{PPh}_{3}\right)_{4}\right]$ gave mixtures from which pure 4 could not be separated. We therefore opted for the alternative route to 4 shown in Scheme 4. Compound 4 a was formed by treatment of 4-(9H-carbazol-9-yl)benzaldehyde with 2,9dimethyl-1,10-phenanthroline-5,6-dione and $\mathrm{NH}_{4} \mathrm{OAc}$; subsequent alkylation of the imidazole gave 4 in $18.3 \%$ yield. The electrospray mass spectra of $\mathbf{3}$ and $\mathbf{4}$ exhibited ions arising from $[\mathrm{M}+\mathrm{H}]^{+}$, and the compounds were characterized by ${ }^{13} \mathrm{C}$ and ${ }^{1} \mathrm{H}$ NMR spectroscopies using COSY, NOESY, HMQC and HMBC techniques. The alkyl chain desymmetrizes the phen unit, giving rise to pairs of signals in both the ${ }^{1} \mathrm{H}$ and ${ }^{13} \mathrm{C}$ NMR spectra for $\mathrm{H} /$ $\mathrm{C}^{\mathrm{B} 3 / \mathrm{B} 8}, \mathrm{H} / \mathrm{C}^{\mathrm{B} 4 / \mathrm{B} 7}$, and $\mathrm{H} / \mathrm{C}^{\mathrm{Me}-p h e n}$. For example, the methyl groups in the ${ }^{1} \mathrm{H}$ NMR spectrum appear at $\delta 3.01$ and $3.07 \mathrm{ppm}$ in 3 , and $\delta$ 2.83 and $2.84 \mathrm{ppm}$ in 4 . In contrast to 4 , the ${ }^{1} \mathrm{H}$ NMR spectrum of precursor $4 \mathrm{a}$ (see Experimental section) reflects the $C_{2}$ symmetry that results from the tautomerism of the imidazole ring.

The synthetic approach to compound $\mathbf{5}$ (Scheme 2) was based on that described in the patent literature for 4,7-bis(4-(diphenylamino)phenyl)-1,10-phenanthroline. ${ }^{40}$ Suzuki coupling of 4,7-dichloro-2,9-dimethyl-1,10-phenanthroline with two equivalents of 4 -( $N, N$-diphenylamino)phenylboronic acid (Scheme 5) gave 5 in moderate yield. The high-resolution electrospray mass spectrum confirmed the presence of the $[\mathrm{M}+\mathrm{H}]^{+}$ion at $m / z=$ 695.3174. Fig. 1 shows the ${ }^{1} \mathrm{H}$ NMR spectrum of 5 which is consistent with a $C_{2}$-symmetric molecule. Protons $\mathrm{H}^{\mathrm{B} 3}$ and $\mathrm{H}^{\mathrm{B} 5}$ were distinguished using the NOESY cross peak between $\mathrm{H}^{\mathrm{Me}}$

(a)<smiles>CCCCOc1ccc(Nc2ccc(OCCCC)cc2)cc1</smiles>

(b)

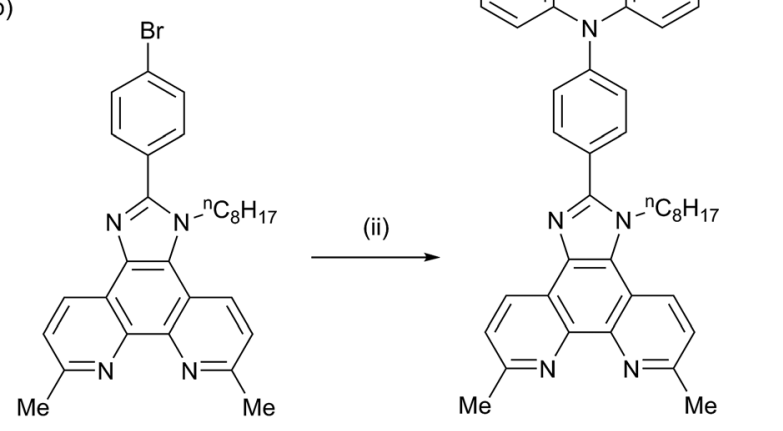

Scheme 3 (a) Synthetic route to 4,4'-di-n-butoxydiphenylamine; conditions (i) $\mathrm{K}_{2} \mathrm{CO}_{3}, \mathrm{Cul}$, L-proline in DMSO, $90^{\circ} \mathrm{C}, 24 \mathrm{~h}$. (b) Conditions for Hartwig-Buchwald coupling to 3: (ii) 4,4'-di-n-butoxydiphenylamine, $\mathrm{NaO}{ }^{t} \mathrm{Bu}$, catalytic $\left[\mathrm{Pd}(\mathrm{dba})_{2}\right] / \mathrm{P}^{t} \mathrm{Bu}$, toluene, reflux $15 \mathrm{~h}$. 
<smiles>Cc1ccc2c(n1)-c1nc(C)ccc1C(=O)C2=O</smiles>

(i)<smiles>COc1ccc2c(n1)c1nc(C)ccc1c1[nH]c(-c3ccc(-n4c5ccccc5c5ccccc54)cc3)nc21</smiles><smiles>CCn1c(-c2ccc(-n3c4ccccc4c4ccccc43)cc2)nc2c3ccc(C)nc3c3nc(C)ccc3c21</smiles>

Scheme 4 Synthetic route to compound 4; conditions (i) excess $\mathrm{NH}_{4} \mathrm{OAc}$, EtOH; (ii) $\mathrm{NaH}$, DMF under $\mathrm{N}_{2}$; (iii) ${ }^{n} \mathrm{C}_{8} \mathrm{H}_{17} \mathrm{Br}$, DMF, $75^{\circ} \mathrm{C}, 15 \mathrm{~h}$.

and $\mathrm{H}^{\mathrm{B} 3} ; \mathrm{H}^{\mathrm{C} 2} / \mathrm{H}^{\mathrm{B} 3}$ and $\mathrm{H}^{\mathrm{C} 2} / \mathrm{H}^{\mathrm{B} 5}$ NOESY cross peaks were used to discriminate between $\mathrm{H}^{\mathrm{C} 2}$ and $\mathrm{H}^{\mathrm{C} 3}$. Assignment of the ${ }^{13} \mathrm{C} \mathrm{NMR}$ spectrum was made using HMBC and HMQC methods.

The solution absorption spectra of the five ligands are compared in Fig. 2. Introduction of the diphenylamino or carbazole units on going from 1 to 2,3 or 4 enhances the

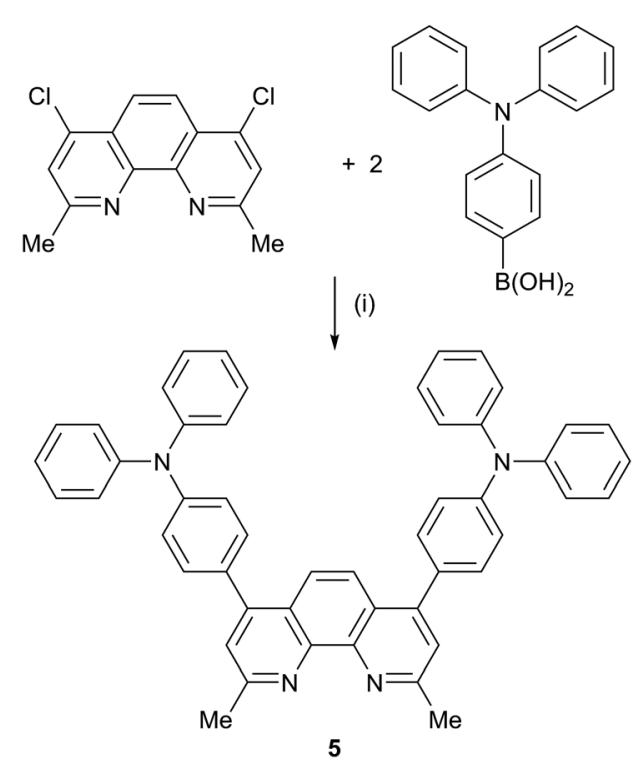

Scheme 5 Synthetic route to compound 5; conditions (i) $\mathrm{K}_{3} \mathrm{PO}_{4}$, catalytic $\left[\mathrm{Pd}(\mathrm{dba})_{2}\right] / \mathrm{P}\left(\mathrm{C}_{6} \mathrm{H}_{11}\right)_{3}, 1,4$-dioxane $/ \mathrm{H}_{2} \mathrm{O}$ (ratio $5: 2$ ), reflux, $95^{\circ} \mathrm{C}, 15 \mathrm{~h}$.

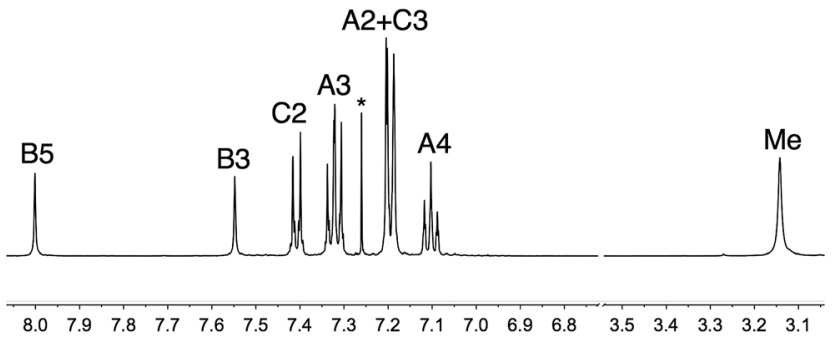

Fig. $1500 \mathrm{MHz}{ }^{1} \mathrm{H}$ NMR spectrum of $5\left(\mathrm{CDCl}_{3}, 295 \mathrm{~K}\right)$. See Scheme 2 for atom labelling; * is residual $\mathrm{CHCl}_{3}$. Chemical shifts are in $\delta / \mathrm{ppm}$.

photoresponse of the ligands in the region between 325 and $400 \mathrm{~nm}$, but the most significant improvement in absorption towards the red-region is observed in compound $\mathbf{5}$ which absorbs down to $\approx 550 \mathrm{~nm}$.

\section{Single crystal structure of 4}

The single crystal structure of $\mathbf{4}$ was determined from crystals grown from a DMSO- $d_{6}$ solution in an NMR tube. The compound crystallizes in the triclinic space group $P \overline{1}$ and the structure is shown in Fig. 3. Bond lengths (caption to Fig. 3) and bond angles are unremarkable, and the phenyl ring containing C19 is twisted through $53.4^{\circ}$ with respect to the plane through the imidazole ring consistent with minimizing inter-ring $\mathrm{H} . . . \mathrm{H}$ contacts. The $n$-octyl chain is folded over the $N$-phenylcarbazole unit (Fig. 3), with an orientation which mimics that in $\mathbf{1}^{26}$ and this leads to close $\mathrm{CH}_{\text {alkyl }} \ldots \pi$ contacts, both to the phenyl spacer $(\mathrm{CH}$...centroid $=3.47 \AA)$ and to the heterocyclic ring of the carbazole $(\mathrm{CH}$...centroid $=3.32 \AA)$. Two packing motifs are of importance. Firstly, carbazole and phen units in adjacent molecules engage in face-to-face $\pi$-stacking interactions, leading to the assembly of chains parallel to the $c$-axis (Fig. $4 \mathrm{a}$ ). As Fig. 3a illustrates, the carbazole and phen units are slipped with respect to one another giving an optimum configuration

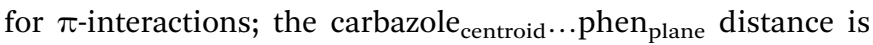
$3.40 \AA$ A. Adjacent chains interact through $\pi$-stacking and this is best described in terms of the quadruple-decker stack shown in Fig. $4 \mathrm{~b}$. The central interaction is between a centrosymmetric pair of $1 H$-phenanthro[9,10- $d]$ imidazole domains (interplane

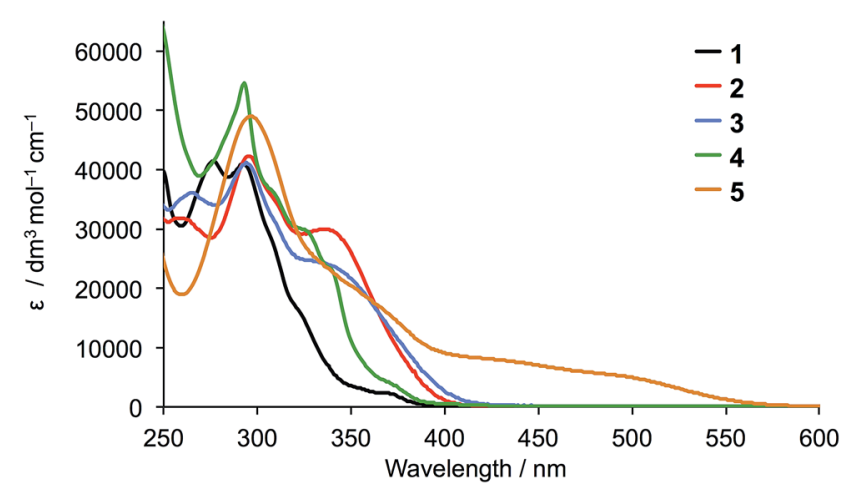

Fig. 2 Solution absorption spectra of ligands $1-5\left(\mathrm{CH}_{2} \mathrm{Cl}_{2}, 1 \times 10^{-5}\right.$ $\mathrm{mol} \mathrm{dm}{ }^{-3}$ ). 


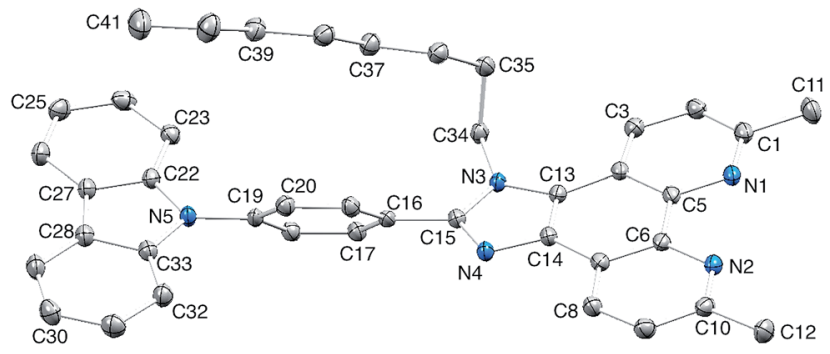

Fig. 3 Crystal structure of 4 with $\mathrm{H}$ atoms omitted and ellipsoids plotted at $50 \%$ probability level. Selected bond lengths: N1-C1 = $1.3250(14), \mathrm{N} 1-\mathrm{C} 5=1.3529(13), \mathrm{N} 2-\mathrm{C} 6=1.3551(14), \mathrm{N} 2-\mathrm{C} 10=$ 1.3271(14), N3-C13 = 1.3892(13), N3-C15 = 1.3783(13), N3-C34 = $1.4644(13), \mathrm{N} 4-\mathrm{C} 14=1.3758(13), \mathrm{N} 4-\mathrm{C} 15=1.3152(14), \mathrm{N} 5-\mathrm{C} 19=$ $1.4216(13), \mathrm{N} 5-\mathrm{C} 22=1.3905(13), \mathrm{N} 5-\mathrm{C} 33=1.3954(13) \AA$.

separation $=3.33 \AA$ ). Extension beyond the quadruple-decker unit is prevented by the $\mathrm{CH} . . \pi$ contacts from the terminal methyl group of the $n$-octyl chain (Fig. 4b).

\section{Synthesis and characterization of homoleptic copper complexes}

The copper(I) complexes $\left[\mathrm{CuL}_{2}\right]\left[\mathrm{PF}_{6}\right]$ with $\mathrm{L}=\mathbf{1}, \mathbf{3 - 5}$ were prepared by dropwise addition of an $\mathrm{MeCN}$ solution of $\left[\mathrm{Cu}(\mathrm{MeCN})_{4}\right]\left[\mathrm{PF}_{6}\right]$ to a solution of the ligand in $\mathrm{CHCl}_{3}$ or $\mathrm{CH}_{2} \mathrm{Cl}_{2}$. The preparation of $\left[\mathrm{Cu}(2)_{2}\right]\left[\mathrm{PF}_{6}\right]$ has previously been reported. ${ }^{26}$ The homoleptic complexes were isolated in 70.5-100\% yield, and in the electrospray mass spectrum of each, the highest mass peak envelope corresponded to the $\left[\mathrm{M}-\mathrm{PF}_{6}\right]^{+}$ion. ${ }^{1} \mathrm{H}$ and ${ }^{13} \mathrm{C}$ NMR spectra were assigned using COSY, NOESY, HMQC and HMBC methods (Fig. S1 $\dagger$ ). Differing

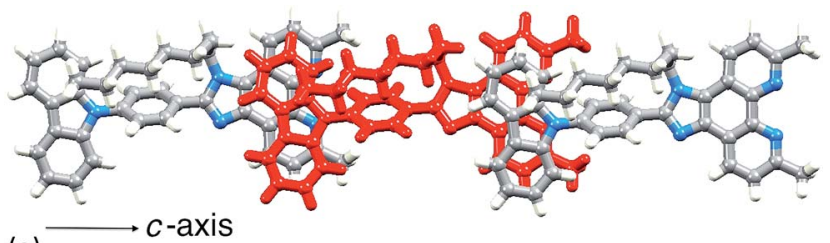

(a)

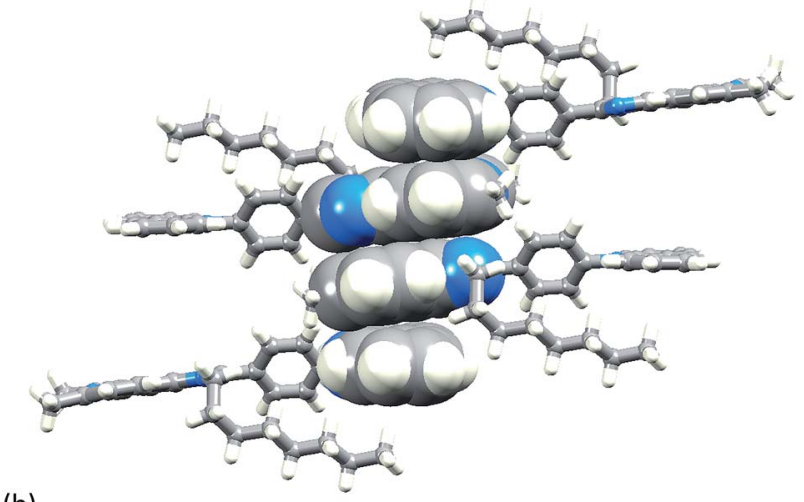

(b)

Fig. 4 (a) In 4 , chains run along the $c$-axis, assembled through $\pi$-stacking interactions between phen and carbazole units. (b) One quadruple-decker $\pi$-stack in 4 .
Ligand 4

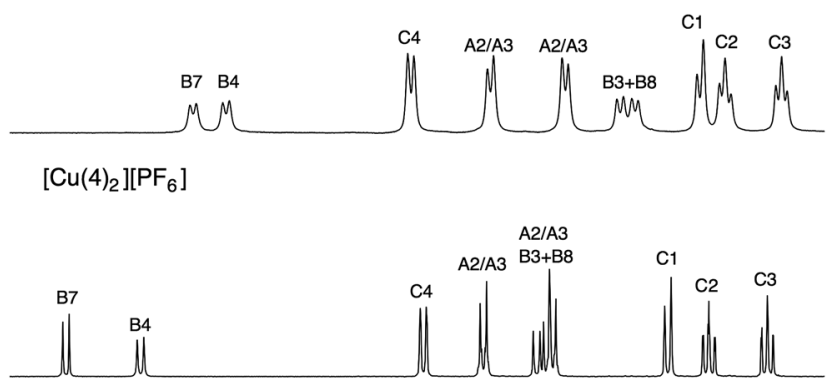

\begin{tabular}{llllllllllllllllllllll}
\hline 9.3 & 9.2 & 9.1 & 9.0 & 8.9 & 8.8 & 8.7 & 8.6 & 8.5 & 8.4 & 8.3 & 8.2 & 8.1 & 8.0 & 7.9 & 7.8 & 7.7 & 7.6 & 7.5 & 7.4 & 7.3
\end{tabular}

Fig. 5 Comparison of the aromatic regions of the $500 \mathrm{MHz}{ }^{1} \mathrm{H}$ NMR spectra of 4 (in DMSO- $d_{6}$ ) and $\left[\mathrm{Cu}(4)_{2}\right]\left[\mathrm{PF}_{6}\right]$ (in $\mathrm{CD}_{3} \mathrm{CN}$ ). See Scheme 2 for atom labelling.

solubility properties of free ligands and complexes precluded the use of common solvents for recording NMR spectra of ligands and copper(I) complexes. Nonetheless, the shifts to higher frequencies for the signals of the phen unit $\left(\mathrm{H}^{\mathrm{B} 3}, \mathrm{H}^{\mathrm{B} 8}\right.$, $\mathrm{H}^{\mathrm{B} 4}$ and $\mathrm{H}^{\mathrm{B} 7}$ ) upon complexation are characteristic, as illustrated for 4 to $\left[\mathrm{Cu}(4)_{2}\right]\left[\mathrm{PF}_{6}\right]$ in Fig. 5 .

The solution absorption spectra of $\left[\mathrm{CuL}_{2}\right]\left[\mathrm{PF}_{6}\right]$ with $\mathrm{L}=\mathbf{1 - 5}$ are shown in Fig. 6. The approximate doubling in the values of the extinction coefficients for the high-energy, ligand-centred absorptions (assigned to $\pi^{*} \leftarrow \pi$ transitions) on going from $\mathrm{L}$ (Fig. 4) to $\left[\mathrm{CuL}_{2}\right]^{+}$(Fig. 6) is consistent with the formation of the homoleptic complexes. The absorption around $470-475 \mathrm{~nm}$ for $\left[\mathrm{Cu}(1)_{2}\right]\left[\mathrm{PF}_{6}\right],\left[\mathrm{Cu}(2)_{2}\right]\left[\mathrm{PF}_{6}\right],\left[\mathrm{Cu}(3)_{2}\right]\left[\mathrm{PF}_{6}\right]$ and $\left[\mathrm{Cu}(4)_{2}\right]\left[\mathrm{PF}_{6}\right]$ arises from metal-to-ligand charge transfer (MLCT) and this undergoes a bathochromic shift to $486 \mathrm{~nm}$ on going to $\left[\mathrm{Cu}(5)_{2}\right]\left[\mathrm{PF}_{6}\right]$. The enhanced spectral response of $\left[\mathrm{Cu}(5)_{2}\right]\left[\mathrm{PF}_{6}\right]$ at wavelengths above $375 \mathrm{~nm}$ is noteworthy in terms of the incorporation of the $\{\mathrm{Cu}(5)\}$ unit in $\mathrm{TiO}_{2}$-bound sensitizers (see later).

The copper(I) complexes are redox active and cyclic voltammograms were recorded in $\mathrm{CH}_{2} \mathrm{Cl}_{2}$ to avoid possible involvement by coordinating solvents such as MeCN. $\left[\mathrm{Cu}(\mathbf{1})_{2}\right]\left[\mathrm{PF}_{6}\right]$ exhibits a reversible oxidation process (Fig. 7) at $+0.48 \mathrm{~V}$

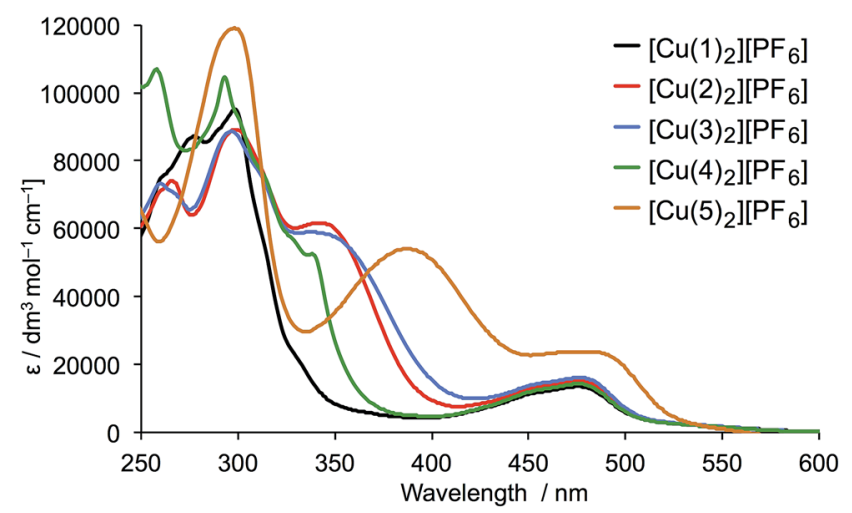

Fig. 6 Solution absorption spectra of complexes $\left[\mathrm{CuL}_{2}\right]\left[\mathrm{PF}_{6}\right]$ for $\mathrm{L}=$ 1-5 $\left(\mathrm{CH}_{2} \mathrm{Cl}_{2}, 1 \times 10^{-5} \mathrm{~mol} \mathrm{dm}{ }^{-3}\right)$. 


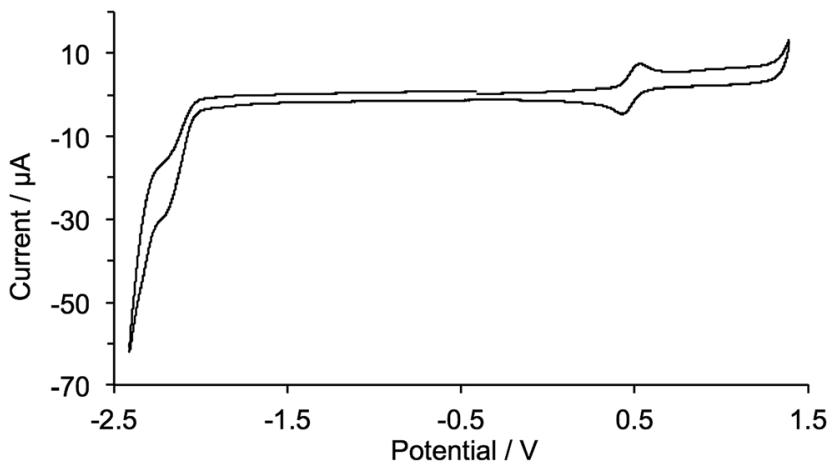

Fig. 7 Cyclic voltammogram of $\left[\mathrm{Cu}(1)_{2}\right]\left[\mathrm{PF}_{6}\right]$ in $\mathrm{CH}_{2} \mathrm{Cl}_{2}$ solution $\left(E_{\mathrm{pc}}-\right.$ $E_{\mathrm{pa}}=74 \mathrm{mV}$ ) with respect to $\mathrm{Fc} / \mathrm{Fc}^{+}$; scan rate $=0.1 \mathrm{~V} \mathrm{~s}^{-1}$.

assigned to the $\mathrm{Cu}^{+} / \mathrm{Cu}^{2+}$ redox couple. The value is close to the reported value of $+0.50 \mathrm{~V}$ for 2,9-dimethyl-1,10-phenanthroline ${ }^{\mathbf{4 1}}$ indicating that the 2-(4-bromophenyl)-1-octyl- $1 H$-imidazo unit in 1 has little effect on the oxidation potential of the copper(I) centre. For $\left[\mathrm{Cu}(2)_{2}\right]\left[\mathrm{PF}_{6}\right],\left[\mathrm{Cu}(3)_{2}\right]\left[\mathrm{PF}_{6}\right],\left[\mathrm{Cu}(4)_{2}\right]\left[\mathrm{PF}_{6}\right]$ and $\left[\mathrm{Cu}(5)_{2}\right]\left[\mathrm{PF}_{6}\right]$, a number of quasi-reversible or irreversible oxidation processes were observed (Fig. S2 $\dagger$ ) consistent with the introduction of the diphenylamino or carbazole functionalizations; these were not investigated in detail. Ligand-based reduction processes are poorly defined (Fig. 7) for all the complexes.

\section{Preparation of DSCs and solid-state absorption spectra of dye- functionalized electrodes}

The $\left[\mathrm{Cu}\left(\mathrm{L}_{\text {anchor }}\right)\left(\mathrm{L}_{\text {ancillary }}\right)\right]^{+}$dyes, in which $\mathrm{L}_{\text {anchor }}$ is the phosphonic acid anchoring ligand $6^{42}$ (Scheme 6) and $\mathrm{L}_{\text {ancillary }}$ is 1$\mathbf{5}$, were assembled on $\mathrm{TiO}_{2}$ electrodes by first soaking them in a solution of $\mathrm{L}_{\text {anchor }}$ followed by immersion in solutions of $\left[\mathrm{Cu}(\mathbf{1})_{2}\right]\left[\mathrm{PF}_{6}\right], \quad\left[\mathrm{Cu}(2)_{2}\right]\left[\mathrm{PF}_{6}\right], \quad\left[\mathrm{Cu}(3)_{2}\right]\left[\mathrm{PF}_{6}\right], \quad\left[\mathrm{Cu}(\mathbf{4})_{2}\right]\left[\mathrm{PF}_{6}\right]$ or $\left[\mathrm{Cu}(5)_{2}\right]\left[\mathrm{PF}_{6}\right]$. Dye-bath concentrations and dipping times were the same for all electrodes. Commercial $\mathrm{TiO}_{2}$ electrodes with or without a scattering layer were used for DSC measurements or solid-state absorption spectroscopy, respectively. Electrodes with the reference dye N719 were prepared by soaking the $\mathrm{TiO}_{2}$ electrodes in solutions of the sensitizer. Although we have previously shown that DSCs (with screen-printed $\mathrm{TiO}_{2}$ ) incorporating $[\mathrm{Cu}(6)(2)]^{+}$perform similarly using either $\mathrm{I}^{-} / \mathrm{I}_{3}{ }^{-}$or $\left[\mathrm{Co}(\mathrm{bpy})_{3}\right]^{2+/ 3+}$ electrolytes, ${ }^{26}$ we chose in the present work to use a standard $\mathrm{I}^{-} / \mathrm{I}_{3}^{-}$electrolyte (see Experimental section).

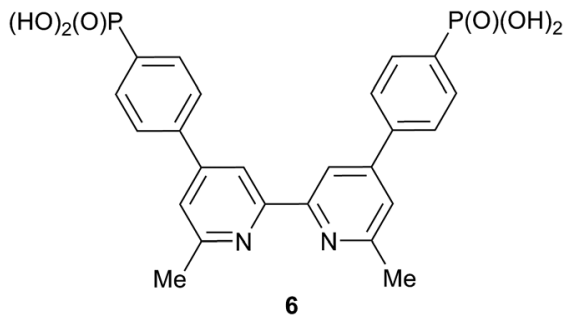

Scheme 6 Structure of the anchoring ligand 6.
Table 1 Performance parameters of masked DSCs with the copper(I) dyes; two DSCs were fabricated for each dye. Data are compared to a DSC containing N719. See Schemes 2 and 6 for ligand structures

\begin{tabular}{llllll}
\hline Dye & $J_{\mathrm{SC}} / \mathrm{mA} \mathrm{cm}^{-2}$ & $V_{\mathrm{OC}} / \mathrm{mV}$ & $\mathrm{ff} / \%$ & $\eta / \%$ & Relative $\eta / \%$ \\
\hline \multicolumn{5}{l}{ On the day of sealing the DSCs } \\
{$[\mathrm{Cu}(\mathbf{6})(\mathbf{1})]^{+}$} & 5.38 & 542 & 71 & 2.08 & 25.8 \\
{$[\mathrm{Cu}(\mathbf{6})(\mathbf{1})]^{+}$} & 5.82 & 547 & 72 & 2.30 & 28.6 \\
{$[\mathrm{Cu}(\mathbf{6})(2)]^{+}$} & 5.41 & 562 & 75 & 2.29 & 28.4 \\
{$[\mathrm{Cu}(\mathbf{6})(2)]^{+}$} & 4.71 & 558 & 73 & 1.92 & 23.9 \\
{$[\mathrm{Cu}(\mathbf{6})(\mathbf{3})]^{+}$} & 4.75 & 523 & 73 & 1.81 & 22.5 \\
{$[\mathrm{Cu}(\mathbf{6})(\mathbf{3})]^{+}$} & 5.65 & 540 & 68 & 2.07 & 25.7 \\
{$[\mathrm{Cu}(\mathbf{6})(\mathbf{4})]^{+}$} & 5.82 & 556 & 73 & 2.35 & 29.1 \\
{$[\mathrm{Cu}(\mathbf{6})(\mathbf{4})]^{+}$} & 5.89 & 562 & 72 & 2.38 & 29.5 \\
{$[\mathrm{Cu}(\mathbf{6})(5)]^{+}$} & 6.81 & 557 & 72 & 2.73 & 33.9 \\
{$[\mathrm{Cu}(\mathbf{6})(5)]^{+}$} & 6.40 & 558 & 73 & 2.62 & 32.5 \\
$\mathrm{~N} 719$ & 17.94 & 642 & 70 & 8.06 & 100.0
\end{tabular}

3 days after sealing the DSCs

$\begin{array}{llllll}{[\mathrm{Cu}(\mathbf{6})(\mathbf{1})]^{+}} & 5.32 & 548 & 71 & 2.06 & 24.3 \\ {[\mathrm{Cu}(\mathbf{6})(\mathbf{1})]^{+}} & 5.57 & 555 & 72 & 2.23 & 26.3 \\ {[\mathrm{Cu}(\mathbf{6})(\mathbf{2})]^{+}} & 4.84 & 570 & 75 & 2.06 & 24.3 \\ {[\mathrm{Cu}(\mathbf{6})(\mathbf{2})]^{+}} & 4.24 & 567 & 72 & 1.74 & 20.4 \\ {[\mathrm{Cu}(\mathbf{6})(\mathbf{3})]^{+}} & 4.47 & 539 & 72 & 1.73 & 20.4 \\ {[\mathrm{Cu}(\mathbf{6})(3)]^{+}} & 5.11 & 546 & 68 & 1.88 & 22.2 \\ {[\mathrm{Cu}(\mathbf{6})(\mathbf{4})]^{+}} & 5.54 & 558 & 72 & 2.22 & 26.1 \\ {[\mathrm{Cu}(\mathbf{6})(\mathbf{4})]^{+}} & 5.26 & 570 & 71 & 2.14 & 25.2 \\ {[\mathrm{Cu}(\mathbf{6})(\mathbf{5})]^{+}} & 6.47 & 567 & 71 & 2.59 & 30.5 \\ {[\mathrm{Cu}(\mathbf{6})(\mathbf{5})]^{+}} & 6.17 & 564 & 73 & 2.54 & 30.0 \\ \mathrm{~N} 719 & 17.77 & 700 & 68 & 8.49 & 100.0\end{array}$

The solid-state absorption spectra of the sensitized electrodes are shown in Fig. 8; Fig. S3† shows photographs of the electrodes, all of which had the same soaking conditions in the dye baths. The values of $\lambda_{\max }$ for the MLCT bands of $\left[\mathrm{Cu}(\mathbf{1})_{2}\right]^{+}$, $\left[\mathrm{Cu}(2)_{2}\right]^{+},\left[\mathrm{Cu}(3)_{2}\right]^{+}$and $\left[\mathrm{Cu}(4)_{2}\right]^{+}$in solution $(470-475 \mathrm{~nm})$ are consistent with those of the on-surface dyes $[\mathrm{Cu}(\mathbf{6})(\mathbf{1})]^{+}$, $[\mathrm{Cu}(6)(2)]^{+},[\mathrm{Cu}(6)(3)]^{+}$and $[\mathrm{Cu}(6)(4)]^{+}(466-469 \mathrm{~nm})$. Pleasingly, the enhanced absorption between 375-600 $\mathrm{nm}$ shown by $\left[\mathrm{Cu}(5)_{2}\right]^{+}$compared to the other homoleptic dyes in solution (Fig. 6) is also observed for the surface-anchored $[\mathrm{Cu}(6)(5)]^{+}$ (Fig. 8). However, none of the dyes absorbs as far into the red as N719.

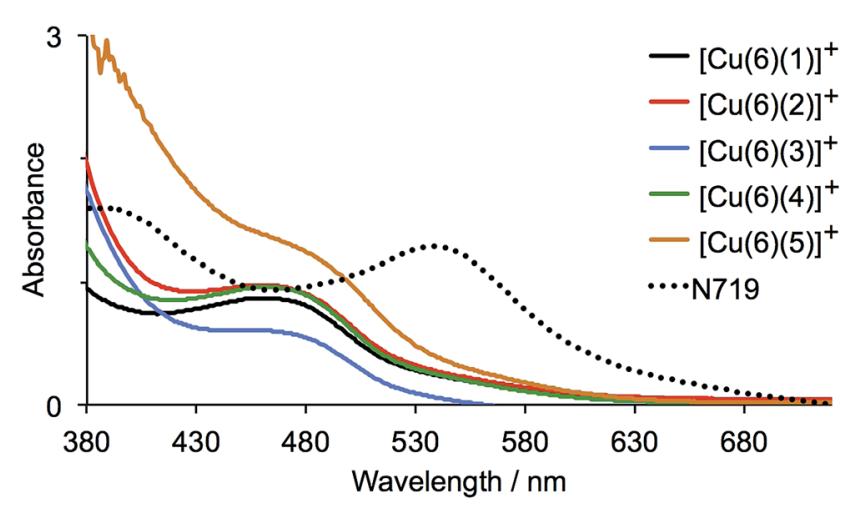

Fig. 8 Solid-state absorption spectra of dye-functionalized $\mathrm{TiO}_{2}$ electrodes. 


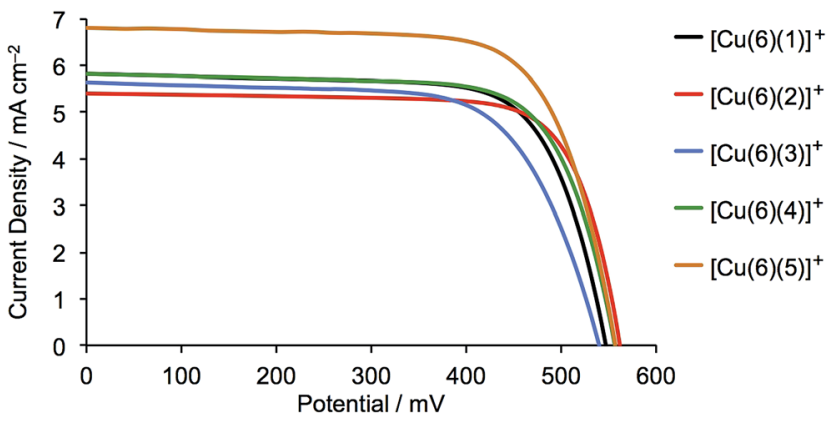

Fig. $9 \mathrm{~J}-V$ curves for DSCs containing the sensitizers $[\mathrm{Cu}(6)(1)]^{+}$ $[\mathrm{Cu}(6)(2)]^{+},[\mathrm{Cu}(6)(3)]^{+},[\mathrm{Cu}(6)(4)]^{+}$and $[\mathrm{Cu}(6)(5)]^{+}$; see also Fig. $54 \dagger$ Data were recorded on the day of DSC fabrication.

\section{DSC performances}

Table 1 gives the performance parameters for duplicate DSCs containing $[\mathrm{Cu}(\mathbf{6})(\mathbf{1})]^{+},[\mathrm{Cu}(\mathbf{6})(2)]^{+},[\mathrm{Cu}(\mathbf{6})(3)]^{+},[\mathrm{Cu}(\mathbf{6})(\mathbf{4})]^{+}$and $[\mathrm{Cu}(\mathbf{6})(5)]^{+}$on the day of assembly (day 0) and after 3 days. All DSCs show similar fill factor (ff) values. Values of the opencircuit voltage $\left(V_{\mathrm{OC}}\right)$ for all the copper(I) dyes lie in the range 523-562 $\mathrm{mV}$ on day 0 and show a small gain over a 3 day ageing period (Table 1). Improved performance over time is a known phenomenon for copper(I)-containing dyes combined with an $\mathrm{I}^{-} / \mathrm{I}_{3}{ }^{-}$electrolyte; ${ }^{24}$ it has also been reported for ruthenium(II) dyes and appears to arise from disaggregation and reorganization of the surface-bound dye molecules. ${ }^{43}$ For DSCs with $[\mathrm{Cu}(6)(\mathbf{1})]^{+},[\mathrm{Cu}(\mathbf{6})(2)]^{+},[\mathrm{Cu}(6)(3)]^{+},[\mathrm{Cu}(\mathbf{6})(4)]^{+}$and $[\mathrm{Cu}(6)(5)]^{+}$, the improved $V_{\mathrm{OC}}$ values are not complemented by an increase in the short-circuit current density $\left(J_{\mathrm{SC}}\right)$ over time, and the overall efficiencies $(\eta)$ remain similar or decrease from day 0 to day 3 (Table 1).

An important point is that these studies can be used to validate comparisons between DSC data from our laboratory where we use both commercial electrodes and those made inhouse. Images obtained using scanning electron microscopy confirm that the $\approx 9 \mu \mathrm{m}$ thickness of the transparent layer of a commercial electrode $^{44}$ corresponds to 4-layers of in-house

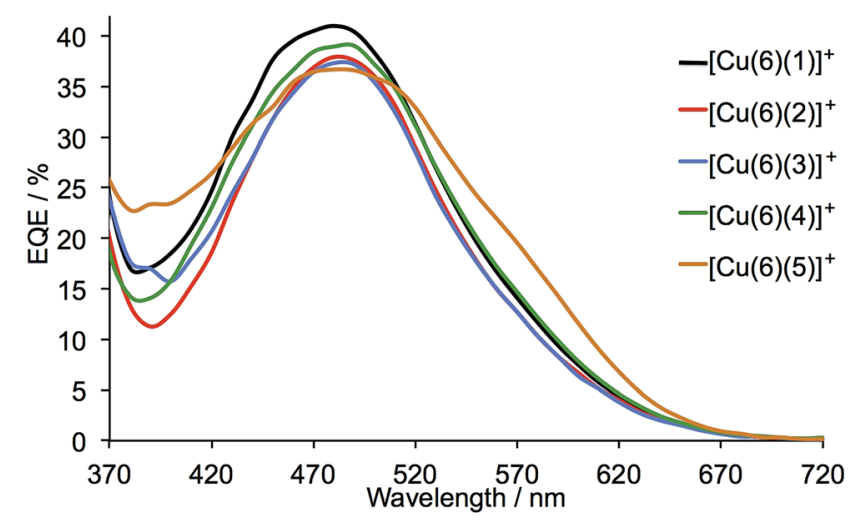

Fig. 10 EQE spectra of DSCs containing the sensitizers $[\mathrm{Cu}(6)(1)]^{+}$, $[\mathrm{Cu}(6)(2)]^{+},[\mathrm{Cu}(6)(3)]^{+},[\mathrm{Cu}(6)(4)]^{+}$and $[\mathrm{Cu}(6)(5)]^{+}$; see also Fig. S5. $\uparrow^{\top}$ The spectra were recorded 3 days after cell assembly.
Table 2 EQE maxima for two independent sets of DSCs containing dyes $\left[\mathrm{Cu}(6)\left(\mathrm{L}_{\text {ancillary }}\right)\right]^{+}$with $\mathrm{L}_{\text {ancillary }}=1-5$ measured 3 days after cell fabrication

\begin{tabular}{lll}
\hline Anchored dye & $\mathrm{EQE}_{\max } / \%$ & $\lambda_{\text {max }} / \mathrm{nm}$ \\
\hline$[\mathrm{Cu}(\mathbf{6})(\mathbf{1})]^{+}$ & 41.0 & 480 \\
{$[\mathrm{Cu}(\mathbf{6})(\mathbf{1})]^{+}$} & 39.4 & 480 \\
{$[\mathrm{Cu}(\mathbf{6})(\mathbf{2})]^{+}$} & 37.9 & 480 \\
{$[\mathrm{Cu}(\mathbf{6})(\mathbf{2})]^{+}$} & 35.3 & 480 \\
{$[\mathrm{Cu}(\mathbf{6})(\mathbf{3})]^{+}$} & 37.3 & 480 \\
{$[\mathrm{Cu}(\mathbf{6})(\mathbf{3})]^{+}$} & 34.7 & 480 \\
{$[\mathrm{Cu}(\mathbf{6})(\mathbf{4})]^{+}$} & 39.0 & 480 \\
{$[\mathrm{Cu}(\mathbf{6})(\mathbf{4})]^{+}$} & 39.1 & 480 \\
{$[\mathrm{Cu}(6)(5)]^{+}$} & 36.6 (sh. 19.5) & 490 (sh. 570) \\
{$[\mathrm{Cu}(\mathbf{6})(\mathbf{5})]^{+}$} & 37.1 (sh. 19.5) & 490 (sh. 570) \\
$\mathrm{N} 719$ & 74.4 & 530
\end{tabular}

screen printed $\mathrm{TiO}_{2}$. The values of $J_{\mathrm{SC}}, V_{\mathrm{OC}}$, ff and $\eta$ of the DSCs with $[\mathrm{Cu}(\mathbf{6})(2)]^{+}$(Table 1) using commercial electrodes with scattering layer and an $\mathrm{I}^{-} / \mathrm{I}_{3}{ }^{-}$electrolyte, compare favourably with parameters $\left(J_{\mathrm{SC}}=5.11 \mathrm{~mA} \mathrm{~cm}{ }^{-2}, V_{\mathrm{OC}}=574 \mathrm{mV}, \mathrm{ff}=\right.$ $71 \%, \eta=2.08 \%$ ) for DSCs containing an $\mathrm{I}^{-} / \mathrm{I}_{3}{ }^{-}$electrolyte with the dye $[\mathrm{Cu}(6)(2)]^{+}$anchored on 4-layer screen-printed electrodes post-treated with $40 \mathrm{mmol} \mathrm{dm}{ }^{-3} \mathrm{H}_{2} \mathrm{O}-\mathrm{TiCl}_{4}{ }^{26}$

The current density/potential $(J-V)$ curves recorded on the day of device fabrication are shown in Fig. $44 ; \dagger J-V$ curves for the best performing device from each pair of duplicate DSCs are displayed in Fig. 9. The DSCs sensitized with $[\mathrm{Cu}(6)(5)]^{+}$ outperform the other solar cells, the main contributing factor being enhanced $J_{\mathrm{SC}}$ values. This is consistent with extended light absorption towards the red for complexes containing $\mathbf{5}$ (Fig. 8) and is confirmed by the higher external quantum efficiencies (EQE) of the DSCs. EQE spectra for all devices are shown Fig. S5, $\dagger$ and Fig. 10 depicts the spectra for the best performing DSC of each pair; values of $\mathrm{EQE}_{\max }$ and $\lambda_{\max }$ are given in Table 2 . Although the values of $\mathrm{EQE}_{\max }$ for $[\mathrm{Cu}(\mathbf{6})(\mathbf{1})]^{+}$ and $[\mathrm{Cu}(6)(4)]^{+}$are higher than for $[\mathrm{Cu}(6)(5)]^{+}$(Table 2), the extension of the EQE spectrum of the DSCs with $[\mathrm{Cu}(\mathbf{6})(5)]^{+}$to longer wavelengths (Fig. 10 and $\mathrm{S} 5 \dagger$ ) leads to higher $J_{\mathrm{SC}}$ values with respect to DSCs with the other dyes. Fig. S6† shows a comparison of the EQE spectra of duplicate DSCs containing $[\mathrm{Cu}(6)(5)]^{+}$with the EQE spectrum of an N719 sensitized DSC, and demonstrates the origins of the lower values of $J_{\mathrm{SC}}$ for the copper(I) dye versus the ruthenium(II) reference dye.

\section{Conclusions}

We have reported the synthesis and characterization of a series of homoleptic $\left[\mathrm{Cu}(\mathrm{L})_{2}\right]\left[\mathrm{PF}_{6}\right]$ complexes in which $\mathrm{L}$ is a 2,9-dimethyl-1,10-phenanthroline substituted in either the 5,6-positions with a peripherally-functionalized imidazole unit or in the 4,7-positions with electron-donating 4-(diphenylamino)phenyl groups. The solution absorption spectrum of $\left[\mathrm{Cu}(5)_{2}\right]\left[\mathrm{PF}_{6}\right]$ exhibits a greater spectral response above $375 \mathrm{~nm}$ than those of $\left[\mathrm{Cu}(\mathbf{1})_{2}\right]\left[\mathrm{PF}_{6}\right],\left[\mathrm{Cu}(2)_{2}\right]\left[\mathrm{PF}_{6}\right],\left[\mathrm{Cu}(3)_{2}\right]\left[\mathrm{PF}_{6}\right]$ and $\left[\mathrm{Cu}(4)_{2}\right]\left[\mathrm{PF}_{6}\right]$. The heteroleptic dyes $[\mathrm{Cu}(\mathbf{6})(\mathrm{L})]^{+}$were assembled in a stepwise manner on $\mathrm{TiO}_{2}$ electrodes, and solid-state 
absorption spectra confirmed enhanced absorption between 375-600 nm for $[\mathrm{Cu}(\mathbf{6})(5)]^{+}$compared to $[\mathrm{Cu}(\mathbf{6})(\mathbf{1})]^{+},[\mathrm{Cu}(\mathbf{6})(2)]^{+}$, $[\mathrm{Cu}(6)(3)]^{+}$and $[\mathrm{Cu}(6)(4)]^{+}$. Comparison of the performances of DSCs containing $[\mathrm{Cu}(\mathbf{6})(2)]^{+},[\mathrm{Cu}(6)(3)]^{+}$and $[\mathrm{Cu}(\mathbf{6})(4)]^{+}$with those with $[\mathrm{Cu}(\mathbf{6})(\mathbf{1})]^{+}$suggests only a marginal influence of the diphenylamine or carbazole hole-transporting domains in 5,6-substituted phenanthroline dyes. In ancillary ligand $\mathbf{5}$, the 4-(diphenylamino)phenyl hole-transporting units are introduced directly into the 4- and 7-positions of the phen unit, and this combined with a phosphonic anchoring domain in $[\mathrm{Cu}(6)(5)]^{+}$leads to the best performing DSCs of those investigated. Although the values of $\mathrm{EQE}_{\max }$ for $[\mathrm{Cu}(\mathbf{6})(\mathbf{1})]^{+}$and $[\mathrm{Cu}(6)(4)]^{+}$exceed that of $[\mathrm{Cu}(6)(5)]^{+}$, the extension of the EQE spectrum of the DSCs with $[\mathrm{Cu}(6)(5)]^{+}$towards the red-end of the spectrum results in higher $J_{\mathrm{SC}}$ values with respect to DSCs with the other dyes. We are currently exploring the effects on DSC performance of introducing other substituents in the 4,7-positions of 2,9-dimethyl-1,10-phenanthrolines used as ancillary ligands in heteroleptic copper(I) sensitizers, and are also focusing on electrolyte optimization.

\section{Acknowledgements}

We acknowledge financial support from the European Research Council (Advanced Grant 267816 LiLo), the Swiss National Science Foundation (Grant 200020_144500) and the University of Basel.

\section{Notes and references}

1 B. O'Regan and M. Grätzel, Nature, 1991, 353, 737.

2 G. C. Vougioukalakis, A. I. Philippopoulos, T. Stergiopoulos and P. Falaras, Coord. Chem. Rev., 2011, 255, 2602.

3 A. Mishra, M. K. R. Fischer and P. Bäuerle, Angew. Chem., Int. Ed., 2009, 48, 2474.

4 L.-L. Li and E. W.-G. Diau, Chem. Soc. Rev., 2013, 42, 291.

5 A. Yella, H.-W. Lee, H. N. Tsao, C. Yi, A. K. Chandiran, M. K. Nazeeruddin, E. W.-G. Diau, C.-Y. Yeh, S. M. Zakeeruddin and M. Grätzel, Science, 2011, 334, 629; M. Zhang, Y. Wang, M. Xu, W. Ma, R. Li and P. Wang, Energy Environ. Sci., 2013, 6, 2944; K. Kakiage, Y. Aoyama, T. Yano, T. Otsuka, T. Kyomen, M. Unno and M. Hanaya, Chem. Commun., 2014, 50, 6379; H. Ozawa, Y. Okuyama and H. Arakawa, ChemPhysChem, 2014, 15, 1201.

6 Z. Lin, N.-G. Park and G. Li, J. Mater. Chem. A, 2015, 3, 8924 and papers in this perovskite-themed issue of the journal.

7 N. J. Jeon, J. H. Noh, W. S. Yang, Y. C. Kim, S. Ryu, J. Seo and S. I. Seok, Science, 2015, 348, 1234.

8 W. S. Yang, J. H. Noh, N. J. Jeon, Y. C. Kim, S. Ryu, J. Seo and S. I. Seok, Nature, 2015, 517, 476.

9 B. Bozic-Weber, E. C. Constable and C. E. Housecroft, Coord. Chem. Rev., 2013, 257, 3089.

10 T. Bessho, E. C. Constable, M. Graetzel, A. Hernandez Redondo, C. E. Housecroft, W. Kylberg, M. K. Nazeeruddin, M. Neuburger and S. Schaffner, Chem. Commun., 2008, 3717; B. Bozic-Weber, E. C. Constable,
C. E. Housecroft, P. Kopecky, M. Neuburger and J. A. Zampese, Dalton Trans., 2011, 12584.

11 N. Alonso-Vante, J.-F. Nierengarten and J.-P. Sauvage, J. Chem. Soc., Dalton Trans., 1994, 1649.

12 F. J. Malzner, S. Y. Brauchli, E. C. Constable, C. E. Housecroft and M. Neuburger, RSC Adv., 2014, 4, 48712.

13 M. Sandroni, L. Favereau, A. Planchat, H. Akdas-Kilig, N. Szuwarski, Y. Pellegrin, E. Blart, H. Le Bozec, M. Boujtita and F. Odobel, J. Mater. Chem. A, 2014, 2, 9944. 14 A. Colombo, C. Dragonetti, M. Magni, D. Roberto, F. Demartin, S. Caramori and C. A. Bignozzi, ACS Appl. Mater. Interfaces, 2014, 6, 13945.

15 See for example: S. Sakaki, T. Kuroki and T. Hamada, J. Chem. Soc., Dalton Trans., 2002, 840; E. C. Constable, A. Hernandez Redondo, C. E. Housecroft, M. Neuburger and S. Schaffner, Dalton Trans., 2009, 6634; B. BozicWeber, E. C. Constable, C. E. Housecroft, M. Neuburger and J. R. Price, Dalton Trans., 2010, 3585; A. Colombo, C. Dragonetti, D. Roberto, A. Valore, P. Biagini and F. Melchiorre, Inorg. Chim. Acta, 2013, 407, 204.

16 M. Mohamkumar, F. Monti, M. Holler, F. Niess, B. DelavauxNicot, N. Armaroli, J.-P. Sauvage and J.-F. Nierengarten, Chem.-Eur. J., 2014, 20, 12083 and references therein.

17 See for example: M. Schmittel and A. Ganz, Chem. Commun., 1997, 999; M. Schmittel, H. Ammon, V. Kalsani, A. Wiegrefe and C. Michel, Chem. Commun., 2002, 2566.

18 M. Sandroni, M. Kayanuma, A. Planchat, N. Szuwarski, E. Blart, Y. Pellegrin, C. Daniel, M. Boujtita and F. Odobel, Dalton Trans., 2013, 10818.

19 A. Hernandez Rendondo, E. C. Constable and C. E. Housecroft, Chimia, 2009, 63, 205.

20 E. Schönhofer, B. Bozic-Weber, C. J. Martin, E. C. Constable, C. E. Housecroft and J. A. Zampese, Dyes Pigm., 2015, 115, 154.

21 N. Hostettler, S. O. Fürer, B. Bozic-Weber, E. C. Constable and C. E. Housecroft, Dyes Pigm., 2015, 116, 124.

22 S. Y. Brauchli, F. J. Malzner, E. C. Constable and C. E. Housecroft, $R S C A d v$., 2015, 5, 48516 and references therein.

23 N. Hostettler, I. A. Wright, B. Bozic-Weber, E. C. Constable and C. E. Housecroft, RSC Adv., 2015, 5, 37906 and references therein.

24 S. Y. Brauchli, F. J. Malzner, E. C. Constable and C. E. Housecroft, $R S C A d v ., 2014$, 4, 62728 and references therein.

25 See for example: Y.-C. Hsu, H. Zheng, J. T. Lin and K. C. Ho, Sol. Energy Mater. Sol. Cells, 2005, 87, 357; J.-F. Yin, D. Bhattacharya, Y.-C. Hsu, C.-C. Tsai, K.-L. Lu, H. C. Lin, J.-G. Chen and K.-C. Ho, J. Mater. Chem., 2009, 19, 7036; S.-H. Fan, A.-G. Zhang, C.-C. Ju, L.-H. Gao and K.-Z. Wang, Inorg. Chem., 2010, 49, 3752; S.-H. Fan, A.-G. Zhang, C.-C. Ju, L.-H. Gao and K.-Z. Wang, Sol. Energy, 2011, 85, 2497; Q.-Y. Yu, J.-F. Huang, Y. Shen, L.-M. Xiao, J.-M. Liu, D.-B. Kuang and C.-Y. Su, RSC Adv., 2013, 3, 19311.

26 B. Bozic-Weber, E. C. Constable, S. O. Fürer, C. E. Housecroft, L. J. Troxler and J. A. Zampese, Chem. Commun., 2013, 49, 7222. 
27 See for example: J. E. Kroeze, N. Hirata, S. Koops, M. K. Nazeeruddin, L. Schmidt-Mende, M. Grätzel and J. R. Durrant, J. Am. Chem. Soc., 2006, 128, 16376; Y. Ooyama and Y. Harima, Eur. J. Org. Chem., 2009, 2903; W. H. Nguyen, C. D. Bailie, J. Burschka, T. Moehl, M. Grätzel, M. D. McGehee and A. Sellinger, Chem. Mater., 2013, 25, 1519; Q. Feng, G. Zhou and Z.-S. Wang, J. Power Sources, 2013, 239, 16; K. Omata, S. Kuwahara, K. Katayama, S. Qing, T. Toyoda, K.-M. Lee and C.-G. Wu, Phys. Chem. Chem. Phys., 2015, 17, 10170 and references therein.

28 R. H. Zheng, H. C. Guo, H. J. Jiang, K. H. Xu, B. B. Liu, W. L. Sun and Z. Q. Shen, Chin. Chem. Lett., 2010, 21, 1270.

29 K. Guzow, M. Czerwińska, A. Ceszlak, M. Kozarzewska, M. Szabelski, C. Czaplewski, A. Łukaszewicz, A. Kubicki and W. Wiczk, Photochem. Photobiol. Sci., 2013, 12, 284.

30 A. F. Larsen and T. Ulven, Org. Lett., 2011, 13, 3546.

31 G. J. Kubas, Inorg. Synth., 1990, 28, 68.

32 M. Shallaiah, Y. C. Rajan and H.-C. Lin, J. Mater. Chem., 2012, 22, 8976.

33 S. A. Odom, K. Lancaster, L. Beverina, K. M. Lefler, N. J. Thompson, V. Coropceanu, J.-L. Brédas, S. R. Marder and S. Barlow, Chem.-Eur. J., 2007, 34, 9637.

34 Bruker Analytical X-ray Systems, Inc., APEX2, version 2 User Manual, M86-E01078, Madison, WI, 2006.
35 P. W. Betteridge, J. R. Carruthers, R. I. Cooper, K. Prout and D. J. Watkin, J. Appl. Crystallogr., 2003, 36, 1487.

36 I. J. Bruno, J. C. Cole, P. R. Edgington, M. K. Kessler, C. F. Macrae, P. McCabe, J. Pearson and R. Taylor, Acta Crystallogr., Sect. B: Struct. Sci., 2002, 58, 389.

37 C. F. Macrae, I. J. Bruno, J. A. Chisholm, P. R. Edgington, P. McCabe, E. Pidcock, L. Rodriguez-Monge, R. Taylor, J. van de Streek and P. A. Wood, J. Appl. Crystallogr., 2008, 41, 466.

38 H. J. Snaith, Energy Environ. Sci., 2012, 5, 6513.

39 H. J. Snaith, Nat. Photonics, 2012, 6, 337.

40 N. Herron, M. A. Guidry, V. Rostovtsev, W. Gao, Y. Wang, Y. Shen and J. A. Merlo, Appl. number PCT/US2009/069184 (International Publication Number WO 2010/075379 A2).

41 M. K. Eggleston, D. R. McMillin, K. S. Koenig and A. J. Pallenberg, Inorg. Chem., 1997, 36, 172.

42 B. Bozic-Weber, S. Y. Brauchli, E. C. Constable, S. O. Fürer, C. E. Housecroft, F. J. Malzner, I. A. Wright and J. A. Zampese, Dalton Trans., 2013, 12293.

43 B. Wenger, M. Grätzel and J.-E. Moser, J. Am. Chem. Soc., 2005, 127, 12150; B. Wenger, M. Grätzel and J.-E. Moser, Chimia, 2005, 59, 123; V. K. Thorsmølle, B. Wenger, J. Teuscher, C. Bauer and J.-E. Moser, Chimia, 2007, 61, 631. 44 S. Y. Brauchli, B. Bozic-Weber, E. C. Constable, N. Hostettler, C. E. Housecroft and J. A. Zampese, RSC Adv., 2014, 4, 34801. 